\title{
EXISTENCE, UNIQUENESS AND EXPLICIT BOUNDS FOR ACOUSTIC SCATTERING BY AN INFINITE LIPSCHITZ BOUNDARY WITH AN IMPEDANCE CONDITION
}

\author{
Thomas Baden-Riess
}

thomas_baden_riess@yahoo.co.uk

\begin{abstract}
We study a boundary value problem for the Helmholtz equation with an impedance boundary condition, in two and three dimensions, modelling the scattering of time harmonic acoustic waves by an unbounded rough surface. Via analysis of an equivalent variational formulation we prove this problem to be well-posed when: i) the boundary has the strong local Lipschitz property and the frequency is small; ii) the rough surface is the graph of a bounded Lipschitz function (with arbitrary frequency). An attractive feature of our results is that the bounds we derive, on the inf-sup constants of the sesquilinear forms, are explicit in terms of the wavenumber $k$, the geometry of the scatterer and the parameters describing the surface impedance.
\end{abstract}

\section{Introduction}

In this paper we study a boundary value problem for the Helmholtz equation with an impedance boundary condition, modelling the scattering of time harmonic acoustic waves by an unbounded rough surface that lies within a finite distance of a plane, due to a source term that is supported within a finite distance of the boundary. Our assumption throughout is that the surface is the graph of a bounded Lipschitz function or, for the case of small wavenumber $k$, simply that the surface possesses the strong local Lipschitz property (defined below). The mathematical problem we study (both the 2D and 3D versions) is a standard model of ground effects in outdoor noise propagation (e.g. [46,3]), in which application the impedance is complex-valued and frequency-dependent, and the $2 \mathrm{D}$ version of this problem is of relevance also in electromagnetic scattering (e.g. [53]).

Our aim in this paper is to prove well-posedness of this impedance boundary value problem, moreover obtaining stability estimates that are expressed explicitly in terms of the wavenumber and parameters describing the geometry and surface impedance. In doing this we will extend the methods and results of [21] in which an equivalent problem but with the simpler Dirichlet boundary condition was studied. Our approach will entail reformulating our boundary value problem as a variational problem on a strip above the rough surface. Thus in terms of style and methodology this paper follows on from [21], which work in turn was inspired, in part, by earlier work for the special (and simpler) diffraction-grating case where the boundary is periodic (a diffraction grating) and the variational problem is formulated in a bounded periodic cell (e.g. [37, 6, 50, 32]).

Rough surface scattering problems are well studied and documented in the literature. The review of Warnick and Chew [54] summarises numerical strategies for dealing with this class of problems. A critical survey of scattering approximations is

https://doi.org/10.5186/aasfm.2020.4540

2010 Mathematics Subject Classification: Primary 78A45; Secondary 65J05, 45B05, 28A80.

Key words: Helmholtz equation, reduced wave equation, rough surface scattering, Rellich identity. 
carried out in the review of Elfouhaily and Guerin [30]. In the review [49], Saillard and Sentenac are interested in formulating rough surface scattering problems from a statistical point of view. In [45] Ogilvy reviews research in this area, again with an emphasis on random rough surfaces and on numerical techniques. See also the books by Voronovich [52], Petit [47], Wilcox [55], Pinel and Bourlier [48], and the review of DeSanto [25].

The above books or review articles, from the physics and engineering literature, focus on practical computation and the many important application areas, but do not provide rigorous mathematical theory. The challenges in proving rigorous wellposedness results are substantial: uniqueness may fail (associated with propagating surface waves), and radiation conditions may be unclear; standard arguments to prove Fredholmness, via establishing that operators are compact perturbations of coercive operators, are no longer applicable when the problem cannot be reformulated on a bounded domain.

In the last twenty years, however, methods for the mathematical analysis of these problems have been developed (in large part initiated by the group around Simon Chandler-Wilde, who supervised the PhD project from which this work emanates), proving existence, uniqueness and continuous dependence on the data for a range of scattering scenarios. These mathematical results were proved initially by employing integral equation methods (see for example $[8,9,16,15,56,11]$ ), using generalised collective compactness arguments that apply only for sufficiently smooth boundaries $\left(C^{1, \alpha}\right.$, for some $\left.0<\alpha \leq 1\right)$, and that require analysis in spaces of bounded continuous functions on the infinite boundary, so that the methods can only work in two dimensions as the integral operators are not well-defined for general 3D rough surfaces and bounded continuous densities. Integral equation methods were extended to 3D problems (the Dirichlet rough surface scattering problem), working in $L^{2}$ spaces, but still with sufficiently smooth boundaries, in $[17,18]$, via Rellich-identity-type arguments. These results were later extended to Lipschitz rough surfaces in the author's $\mathrm{PhD}$ thesis [4], combining the methods of these earlier papers with harmonic-analysis-type estimates for double-layer potential operators on Lipschitz domains [51]. In parallel, in [21], a variational approach was developed to good effect, that established well-posedness for the 3D Dirichlet rough surface scattering problem with minimal constraints on boundary smoothness (e.g. the results apply for $C^{0}$ boundaries), moreover using Rellich-identity-type arguments that give explicitly the dependence of the continuity and inf-sup constants in the sesquilinear form on the wavenumber $k$ and on the maximum elevation of the rough surface.

It is from this latter paper [21] that we will take our lead. Indeed, we note that the results and methods of [21] have already prompted significant developments of the same methodology to tackle a wider range of rough surface scattering problems, for example acoustic scattering by penetrable rough layers [39], electromagnetic rough surface scattering (e.g. [42, 41]), weighted space methods that allow a wider class of incident fields (including plane wave incidence in 2D) [13], and elastic scattering problems [31]. This paper can be viewed as a development of the same methodology, but in another direction.

Let us turn now to contrasting the current paper with prior work specifically on the impedance problem that is the focus of this paper, in which a solution $u$ to the Helmholtz equation $\Delta u+k^{2} u=g$ is sought, for which the boundary condition $\frac{\partial u}{\partial \nu}=i k \beta u$ holds. Here $k>0$ is the wavenumber, $g$ is the acoustic source term, $\nu$ is the outward unit normal to the boundary and the impedance $\beta$ is a given function 
defined on the boundary. The authors we quote in the following paragraphs all assume, as a minimum, that $\beta$ is bounded (is in $L^{\infty}(\Gamma)$, where $\Gamma$ is the boundary), and that $\operatorname{Re}(\beta) \geq 0$, this being a necessary and sufficient condition that ensures that acoustic energy does not flow out of $\Gamma$ into the domain of propagation.

In [9] Chandler-Wilde showed the impedance problem to be well-posed in 2D when the boundary is flat, with arbitrary $L^{\infty}$ impedance satisfying that $\operatorname{Re}(\beta) \geq \eta>$ 0 , for some $\eta>0$ (so that the boundary is strictly energy-absorbing), including, in the problem formulation, the case of plane wave incidence. The method applied to obtain these results was to reformulate the problem as an equivalent second kind boundary integral equation on the real line; then to prove uniqueness of solution; and then to infer existence of solution by utilizing the results of [7], which established some novel solvability results for integral equations on the real line. In [56] Zhang and ChandlerWilde were able to show, again using boundary integral equation techniques, that the impedance problem is well-posed in $2 \mathrm{D}$ when the boundary is the graph of a bounded $C^{1,1}$ function.

In [11], Chandler-Wilde and Peplow consider a 2D impedance problem when the boundary is flat (and the impedance $\beta$ constant) except for a local perturbation, this perturbation directed out of the half-plane. They prove uniqueness of solution in the case $\operatorname{Re}(\beta)>0$ (the boundary is energy-absorbing) and reformulate the problem as an equivalent boundary integral equation (well-posedness for the corresponding problem for 3D electromagnetics has recently been established in [26]). This 2D configuration, but allowing a local perturbation of the flat boundary that may be partly directed into, partly directed out of the half-plane, has recently been studied in Bao et al [5], in which well-posedness of a variational formulation in a compact region enclosing the local perturbation is established in the case that $\operatorname{Re}(\beta)>0$ and $\operatorname{Im}(\beta) \geq 0$.

In [27] and [28] Durán, Muga and Nédélec look at the impedance problem (in 2D and $3 \mathrm{D}$ respectively) in the special case that the boundary is flat so that the problem domain is a half plane or half space, obtaining unique existence of solution to their problem. In [29] they then extend their results to the case when the boundary is a locally perturbed half-plane, with constant impedance outside the local perturbation. In contrast to the paper [9] and also the work that we present here, their problem set-up differs in that they assume that $\operatorname{Re}(\beta)=0, \operatorname{Im}(\beta)<0$ (the parameter range in which the surface outside the local perturbation can support surface waves). The problem formulation developed in this paper (and those, for example, in [9] and [56]) are ill-posed (uniqueness fails) in this particular parameter regime: the authors of [27] and [28] achieve well-posedness by employing a different radiation condition to the one used here and in [9], adapted to an assumption that the impedance is constant outside the local perturbation and the presence of a surface wave.

On the numerical side, Chandler-Wilde et al. in [14], [20] and [38], obtain solutions to the impedance problem in a half plane, with various restrictions on the impedance $\beta$. They obtain convergence and stability estimates, illustrative of the relevance of the sort of well-posedness results that we prove in this paper to rigorous numerical analysis. A full numerical analysis of a coupled finite and boundary element method for the locally-perturbed impedance half-plane was recently carried out in [5].

The current paper is based on Chapter 3 of the PhD thesis of the author [4]. Compared to the above literature, there are two main novel aspects; in the first place the results apply in both two and three dimensions; and in the second place the boundaries to which our results apply are more general. Specifically, we prove 
the impedance boundary value problem to be well-posed for $\operatorname{Re}(\beta) \geq \eta>0$, when the boundary is the graph of a bounded Lipschitz function, for all wavenumber $k$. Further, for small wavenumber $k$, we establish well-posedness in the more general case when the boundary has the strong local Lipschitz property (and lies within a finite distance of a plane).

The most closely related other work is that of [35]. In that paper $\mathrm{Hu}$ et al. study the generalised impedance boundary value problem, solving the Helmholtz equation with the generalised impedance boundary condition

$$
\frac{\partial u}{\partial \nu}=\operatorname{div}_{\Gamma}\left(\mu \nabla_{\Gamma} u\right)+i k \beta u
$$

for a given parameter $\mu \in \mathbf{C}$, for the case when the rough surface is the graph of a bounded Lipschitz function, and $\operatorname{Re}(\beta)>0, \operatorname{Im}(\beta) \geq 0$. However, crucially, their results do not apply to the special case where $\mu=0$, when (1) reduces to the classical impedance boundary value problem that we study here. Thus there is no overlap in the content of our theorems. Moreover our results hold not only when the boundary is the graph of a Lipschitz function, but also when it merely has the strong local Lipschitz property, provided the frequency is small. In addition, our requirements on the impedance are less restrictive than in [35]. Specifically, unlike in [35], $\beta$ may take values in the range $\operatorname{Re}(\beta)>0, \operatorname{Im}(\beta)<0$, which is the range that normally arises in applications, for example those in outdoor noise propagation (e.g. [19, 3]).

Acknowledgement. The author would very much like to acknowledge the help and guidance of Simon Chandler-Wilde, without whom this work would not have been possible.

\section{The boundary value problem and variational formulation}

In this section we shall define some notation related to the rough surface scattering problem and write down the boundary value problem and equivalent variational formulation that will be analyzed in later sections. We introduce the following notation: for $x=\left(x_{1}, \ldots, x_{n}\right) \in \mathbf{R}^{n}(n=2,3)$ let $\tilde{x}=\left(x_{1}, \ldots, x_{n-1}\right)$ so that $x=\left(\tilde{x}, x_{n}\right)$. For $H \in \mathbf{R}$ let $U_{H}=\left\{x: x_{n}>H\right\}$ and $\Gamma_{H}=\left\{x: x_{n}=H\right\}$. Let $D \subset \mathbf{R}^{n}$ be an open connected set, with boundary $\Gamma$, such that for some constants $f_{-}<f_{+}$it holds that

$$
U_{f_{+}} \subset D \subset U_{f_{-}} .
$$

In order to make sense of boundary integrals, we will require that for some $\mu>0$ and $N \in \mathbf{N}, D$ be an $(L, \mu, N)$ Lipschitz domain, in the sense of the following definition.

Definition 1. Given $L \in \mathbf{R}, \mu>0$ and $N \in \mathbf{N}$, the set $\Omega$ is said to be an $(L, \mu, N)$ Lipschitz domain if there exists a locally finite open cover $\left\{O_{j}\right\}_{j \in J}$ of $\Gamma$, such that:

i) for each $y \in \Gamma$, the open ball of radius $\mu$ and centre $y$ is a subset of $O_{i}$, for some $i \in J$;

ii) for each $j \in J, O_{j} \cap \Omega=O_{j} \cap \Omega_{j}$, where $\Omega_{j}$ is, after a rotation, the epigraph of a Lipschitz function $f: \mathbf{R}^{n-1} \rightarrow \mathbf{R}$ such that

$$
|f(\tilde{x})-f(\tilde{y})| \leq L|\tilde{x}-\tilde{y}|, \quad \tilde{x}, \tilde{y} \in \mathbf{R}^{n-1} ;
$$

iii) every collection of $N+1$ of the sets $O_{j}$ has empty intersection. 
In fact we will mainly be concerned with the case when $\Gamma$ is the graph of a Lipschitz function:

$$
\Gamma:=\left\{\left(\tilde{x}, x_{n}\right): x_{n}=f(\tilde{x}), \tilde{x} \in \mathbf{R}^{n-1}\right\},
$$

where $f: \mathbf{R}^{n-1} \rightarrow \mathbf{R}$ satisfies (2), in which case $D$ is an $(L, \mu, 1)$ Lipschitz domain, for all $\mu>0$.

Remark 2.1. It is shown in Adams [1] that Definition 1 is equivalent to $\Omega$ having the 'strong local Lipschitz property' as defined in [1]. As Adams remarks, in the case that $\Omega$ is bounded, Definition 1 reduces to the standard definition of a Lipschitz domain.

We denote by $\nu$ the outward unit normal to $D$, which exists almost everywhere by Rademacher's theorem. The variational problem will be posed on the open set $S_{H}:=D \backslash \overline{U_{H}}$, for some $H \geq f_{+}+\mu$, so that $S_{H}$ will be an $(L, \mu, N+1)$ Lipschitz domain.

We will refer to any function $f: \mathbf{R}^{n-1} \rightarrow \mathbf{R}$ that satisfies (2) as a Lipschitz function with Lipschitz constant $L$. Moreover we introduce the notation

$$
J_{f}(\tilde{x})=\sqrt{1+\left|\nabla_{\tilde{x}} f(\tilde{x})\right|^{2}}, \quad \tilde{x} \in \mathbf{R}^{n-1},
$$

and define $L^{\prime}=\sqrt{1+L^{2}}$, so that $J_{f} \leq L^{\prime}$.

For a given source function $g \in L^{2}(D)$, whose support lies within a finite distance of the boundary $\Gamma$, we will look for a weak solution $u$ of the Helmholtz equation

$$
\Delta u+k^{2} u=g \text { in } D,
$$

where $k>0$ is the wavenumber, and with an impedance condition

$$
\frac{\partial u}{\partial \nu}=i k \beta u \text { on } \Gamma,
$$

where $\beta \in L^{\infty}(\Gamma)$ is a given function of the boundary.

To complete the problem formulation a radiation condition is required. In this paper we make use of the so-called upward propagating radiation condition (UPRC). To state this we introduce the fundamental solution to the Helmholtz equation (4). This is $\Phi$, given by

$$
\Phi(x, y)= \begin{cases}\frac{\mathrm{i}}{4} H_{0}^{(1)}(k|x-y|), & n=2 \\ \frac{\exp (\mathrm{i} k|x-y|)}{4 \pi|x-y|}, & n=3\end{cases}
$$

for $x, y \in \mathbf{R}^{n}, x \neq y$, where $H_{0}^{(1)}$ is the Hankel function of the first kind of order zero. $\Phi(x, y)$ is a solution to the Helmholtz equation (4) in the special case when $D=\mathbf{R}^{n}$ and $g=-\delta_{y}$, a point source located at $y \in \mathbf{R}^{n}$. The UPRC then states that

$$
u(x)=2 \int_{\Gamma_{H}} \frac{\partial \Phi(x, y)}{\partial x_{n}} u(y) d s(y), \quad x \in U_{H},
$$

for all $H$ such that the support of $g$ is contained in $S_{H}$.

The UPRC was proposed in [10]. In the case that the wavenumber $k$ has imaginary part, one can derive this representation for the solution of the Helmholtz equation in $U_{H}$, under mild assumptions on the growth of the solution at infinity: see [9].

In the case that $\left.u\right|_{\Gamma_{H}} \in L^{2}\left(\Gamma_{H}\right)$ we can rewrite (6) in terms of the Fourier transform of $\left.u\right|_{\Gamma_{H}}$. For $\phi \in L^{2}\left(\Gamma_{H}\right)$, which we identify with $L^{2}\left(\mathbf{R}^{n-1}\right)$, we denote by 
$\hat{\phi}=\mathcal{F} \phi$ the Fourier transform of $\phi$ which we define by

$$
\mathcal{F} \phi(\xi)=(2 \pi)^{-(n-1) / 2} \int_{\mathbf{R}^{n-1}} \exp (-\mathrm{i} \tilde{x} \cdot \xi) \phi(\tilde{x}) d \tilde{x}, \quad \xi \in \mathbf{R}^{n-1} .
$$

Our choice of normalization of the Fourier transform ensures that $\mathcal{F}$ is a unitary operator on $L^{2}\left(\mathbf{R}^{n-1}\right)$, so that, for $\phi, \psi \in L^{2}\left(\mathbf{R}^{n-1}\right)$,

$$
\int_{\mathbf{R}^{n-1}} \phi \bar{\psi} d \tilde{x}=\int_{\mathbf{R}^{n-1}} \hat{\phi} \overline{\hat{\psi}} d \xi
$$

If $F_{H}:=\left.u\right|_{\Gamma_{H}} \in L^{2}\left(\Gamma_{H}\right)$ then (see [12,2] in the case $n=2$ ), (6) can be rewritten as (9) $u(x)=\frac{1}{(2 \pi)^{(n-1) / 2}} \int_{\mathbf{R}^{n-1}} \exp \left(\mathrm{i}\left[\left(x_{n}-H\right) \sqrt{k^{2}-\xi^{2}}+\tilde{x} \cdot \xi\right]\right) \hat{F}_{H}(\xi) d \xi, \quad x \in U_{H}$.

In this equation $\sqrt{k^{2}-\xi^{2}}=\mathrm{i} \sqrt{\xi^{2}-k^{2}}$, when $|\xi|>k$.

Equation (9) is a representation for $u$, in the upper half-plane $U_{H}$, as a superposition of upward propagating homogeneous and inhomogeneous plane waves. A requirement that (9) holds is commonly used (e.g. [25]) as a formal radiation condition in the physics and engineering literature on rough surface scattering. The meaning of (9) is clear when $F_{H} \in L^{2}\left(\mathbf{R}^{n-1}\right)$ so that $\hat{F}_{H} \in L^{2}\left(\mathbf{R}^{n-1}\right)$; indeed the integral (9) exists in the Lebesgue sense for all $x \in U_{H}$. Arens and Hohage [2] have explained, in the case $n=2$, in what precise sense (9) can be understood when $F_{H} \in B C\left(\Gamma_{H}\right)$ so that $\hat{F}_{H}$ must be interpreted as a tempered distribution. Arens and Hohage also show the equivalence of this radiation condition with another known as the Pole Condition.

We are now in a position to state our boundary value problem.

The Boundary Value Problem. Let $D$ be an $(L, \mu, N)$ Lipschitz domain for some $L>0, \mu>0$ and $N \in \mathbf{N}$. Given $g \in L^{2}(D)$, whose support lies in $S_{H}$ for some $H \geq f_{+}+\mu$, and given $\beta \in L^{\infty}(\Gamma)$, find $u: D \rightarrow \mathbf{C}$ such that $\left.u\right|_{S_{a}} \in H^{1}\left(S_{a}\right)$ for every $a \geq f_{+}+\mu$,

$$
\Delta u+k^{2} u=g \text { in } D, \quad \frac{\partial u}{\partial \nu}=i k \beta u \text { on } \Gamma,
$$

in a distributional sense (see (13) below), and the radiation condition (9) holds with $F_{H}=\left.u\right|_{\Gamma_{H}}$.

Remark 2.2. We note that the solutions of the above problem do not depend on the choice of $H$. Precisely, if $u$ is a solution to the above problem for one value of $H \geq f_{+}+\mu$ for which $\operatorname{supp}(g) \subset \overline{S_{H}}$, then $u$ is a solution for all $H \geq f_{+}+\mu$ with this property. To see that this is true we make analogous arguments to those used in Remark 2.1 of [21].

It will be convenient to work with wavenumber-dependent norms. We will equip the standard Sobolev space $H^{1}\left(S_{H}\right)$ with the $k$-dependent norm, equivalent to the usual norm, given by

$$
\|v\|_{H^{1}\left(S_{H}\right)}:=\left\{k^{2}\|v\|_{L^{2}\left(S_{H}\right)}^{2}+\|\nabla v\|_{L^{2}\left(S_{H}\right)}^{2}\right\}^{\frac{1}{2}}, \quad v \in H^{1}\left(S_{H}\right) .
$$

Let $\mathcal{D}\left(S_{H}\right):=\left\{\left.v\right|_{S_{H}}: v \in C_{0}^{\infty}\left(\mathbf{R}^{n}\right)\right\}$, so that $\mathcal{D}\left(S_{H}\right)$ is dense in $H^{1}\left(S_{H}\right)$. Let $\gamma_{*}: \mathcal{D}\left(S_{H}\right) \rightarrow L^{2}(\Gamma)$ be defined by $\gamma_{*} \phi=\left.\phi\right|_{\Gamma}$ for $\phi \in \mathcal{D}\left(S_{H}\right)$. Then with $S_{H}$ being an $(L, \mu, N+1)$ Lipschitz domain, it's possible to show (see Lemma 5 below) that $\gamma_{*}$ extends to a bounded linear operator $\gamma_{*}: H^{1}\left(S_{H}\right) \rightarrow L^{2}(\Gamma)$. 
Remark 2.3. Trace results on domains with unbounded boundaries appear to be not well written up in the literature, so we will justify the above statement in the next section. In fact we expect that a stronger result holds, but stating this would require defining fractional Sobolev spaces on the boundary $\Gamma$ of an $(L, \mu, N)$ Lipschitz domain. The above result will be sufficient for our needs.

We now derive a variational formulation of the boundary value problem above. We will identify $\Gamma_{H}:=\left\{x: x_{n}=H\right\}$ with $\mathbf{R}^{n-1}$, and then employ standard fractional Sobolev spaces on $\mathbf{R}^{n-1}$ in order to solve the problem. However, we adopt a wavenumber-dependent norm, equivalent to the usual norm, and reducing to the usual norm if the unit of length measurement is chosen so that $k=1$. Thus $H^{s}\left(\Gamma_{H}\right)$, for $s \in \mathbf{R}$, denotes the completion of $C_{0}^{\infty}\left(\Gamma_{H}\right)$ in the norm $\|\cdot\|_{H^{s}\left(\Gamma_{H}\right)}$ defined by

$$
\|\phi\|_{H^{s}\left(\Gamma_{H}\right)}=\left(\int_{\mathbf{R}^{n-1}}\left(k^{2}+\xi^{2}\right)^{s}|\mathcal{F} \phi(\xi)|^{2} d \xi\right)^{1 / 2} .
$$

We recall [1] that, for all $a>H \geq f_{+}+\mu$, there exist continuous embeddings $\gamma_{+}: H^{1}\left(U_{H} \backslash U_{a}\right) \rightarrow H^{1 / 2}\left(\Gamma_{H}\right)$ and $\gamma_{-}: H^{1}\left(S_{H}\right) \rightarrow H^{1 / 2}\left(\Gamma_{H}\right)$ (the trace operators) such that $\gamma_{ \pm} \phi$ coincides with the restriction of $\phi$ to $\Gamma_{H}$ when $\phi$ is $C^{\infty}$. We recall also that, if $u_{+} \in H^{1}\left(U_{H} \backslash U_{a}\right), u_{-} \in H^{1}\left(S_{H}\right)$, and $\gamma_{+} u_{+}=\gamma_{-} u_{-}$, then $v \in H^{1}\left(S_{a}\right)$, where $v(x):=u_{+}(x), x \in U_{H} \backslash U_{a},:=u_{-}(x), x \in S_{H}$. Conversely, if $v \in H^{1}\left(S_{a}\right)$ and $u_{+}:=\left.v\right|_{U_{H} \backslash U_{a}}, u_{-}:=\left.v\right|_{S_{H}}$, then $\gamma_{+} u_{+}=\gamma_{-} u_{-}$. We introduce the operator $T$, a Dirichlet to Neumann map on $\Gamma_{H}$, defined by

$$
T:=\mathcal{F}^{-1} M_{z} \mathcal{F}
$$

where $M_{z}$ is the operation of multiplying by

$$
z(\xi):= \begin{cases}-\mathrm{i} \sqrt{k^{2}-\xi^{2}} & \text { if }|\xi| \leq k \\ \sqrt{\xi^{2}-k^{2}} & \text { for }|\xi|>k\end{cases}
$$

It was proven in Lemma 2.4 in [21] that $T: H^{1 / 2}\left(\Gamma_{H}\right) \rightarrow H^{-1 / 2}\left(\Gamma_{H}\right)$ is bounded with $\|T\|=1$.

Since we will make frequent use of it in this paper, we now recall the following lemma from [21], which describes properties of $u$, defined by (9).

Lemma 2. If (9) holds, with $F_{H} \in H^{1 / 2}\left(\Gamma_{H}\right)$, then $u \in H^{1}\left(U_{H} \backslash U_{a}\right) \cap C^{2}\left(U_{H}\right)$, for every $a>H$,

$$
\Delta u+k^{2} u=0 \text { in } U_{H},
$$

$\gamma_{+} u=F_{H}$, and

$$
\int_{\Gamma_{H}} \bar{v} T \gamma_{+} u d s+k^{2} \int_{U_{H}} u \bar{v} d x-\int_{U_{H}} \nabla u \cdot \nabla \bar{v} d x=0, \quad v \in C_{0}^{\infty}(D) .
$$

Moreover, for all $a>H$, where $F_{a} \in H^{1 / 2}\left(\Gamma_{a}\right)$ denotes the restriction of $u$ to $\Gamma_{a}$, (9) holds with $H$ replaced by $a$.

We now derive a variational formulation of the boundary value problem making use of Lemma 2. Suppose that $u$ satisfies the boundary value problem and recall that $D$ is an $(L, \mu, N)$ Lipschitz domain. Then $\left.u\right|_{S_{a}} \in H^{1}\left(S_{a}\right)$ for every $a \geq f_{+}+\mu$, and, by definition, since (10) holds in a distributional sense,

$$
\int_{D}\left(g \bar{v}+\nabla u \cdot \nabla \bar{v}-k^{2} u \bar{v}\right) d x-\int_{\Gamma} i k \beta \gamma_{*} u \bar{v} d s=0, \quad v \in C_{0}^{\infty}\left(\mathbf{R}^{n}\right) .
$$


Defining $w:=\left.u\right|_{S_{H}}$, and applying Lemma 2 it follows that

$$
\int_{S_{H}}\left(g \bar{v}+\nabla w \cdot \nabla \bar{v}-k^{2} w \bar{v}\right) d x+\int_{\Gamma_{H}} \bar{v} T \gamma_{-} w d s-\int_{\Gamma} i k \beta \gamma_{*} w \bar{v} d s=0, v \in \mathcal{D}\left(S_{H}\right)
$$

From the denseness of $\mathcal{D}\left(S_{H}\right)$ in $H^{1}\left(S_{H}\right)$, and the continuity of $\gamma_{-}$and $\gamma_{*}$, it follows that this equation holds for all $v \in H^{1}\left(S_{H}\right)$.

We let $\|\cdot\|_{2}$ and $(\cdot, \cdot)$ denote the norm and scalar product on $L^{2}\left(S_{H}\right)$ so that $\|v\|_{2}=\sqrt{\int_{S_{H}}|v|^{2} d x}$ and

$$
(u, v)=\int_{S_{H}} u \bar{v} d x
$$

and define the sesquilinear form $c: H^{1}\left(S_{H}\right) \times H^{1}\left(S_{H}\right) \rightarrow \mathbf{C}$ by

$$
c(u, v)=(\nabla u, \nabla v)-k^{2}(u, v)+\int_{\Gamma_{H}} \gamma_{-} \bar{v} T \gamma_{-} u d s-\int_{\Gamma} i k \beta \gamma_{*} u \gamma_{*} \bar{v} d s .
$$

Then we have shown that if $u$ satisfies the boundary value problem then $w:=\left.u\right|_{S_{H}}$ is a solution of the following variational problem: find $u \in H^{1}\left(S_{H}\right)$ such that

$$
c(u, v)=-(g, v), \quad v \in H^{1}\left(S_{H}\right) .
$$

Conversely, suppose that $w$ is a solution to the variational problem and define $u(x)$ to be $w(x)$ in $S_{H}$, and to be the right hand side of (9) in $U_{H}$, with $F_{H}:=\gamma_{-} w$. Then, by Lemma $2, u \in H^{1}\left(U_{H} \backslash \overline{U_{a}}\right)$ for every $a>H$, with $\gamma_{+} u=F_{H}=\gamma_{-} w$. Thus $\left.u\right|_{S_{a}} \in H^{1}\left(S_{a}\right)$ for all $a \geq f_{+}+\mu$. From (12) and (14), it follows that (13) holds, so $u$ satisfies $(10)$.

We have thus proved the following theorem.

Theorem 3. If $u$ is a solution of the boundary value problem then $\left.u\right|_{S_{H}}$ satisfies the variational problem (16). Conversely, if $u$ satisfies the variational problem, $F_{H}:=$ $\gamma_{-} u$, and the definition of $u$ is extended to $D$ by setting $u(x)$ equal to the right hand side of (9), for $x \in U_{H}$, then the extended function satisfies the boundary value problem, with $g$ extended by zero from $S_{H}$ to $D$.

\section{Analysis of the variational problem for low frequency}

Let $H^{1}\left(S_{H}\right)^{*}$ denote the dual space of $H^{1}\left(S_{H}\right)$, i.e. the space of continuous antilinear functionals on $H^{1}\left(S_{H}\right)$. Then our analysis of the variational problem will also apply to the following slightly more general situation: given $\mathcal{G} \in H^{1}\left(S_{H}\right)^{*}$ find $u \in H^{1}\left(S_{H}\right)$ such that

$$
c(u, v)=\mathcal{G}(v), \quad v \in H^{1}\left(S_{H}\right) .
$$

We define the dimensionless wavenumber

$$
\kappa=k\left(H-f_{-}\right),
$$

and assume throughout that $\beta \in L^{\infty}(\Gamma)$ is such that

$$
-\frac{\pi}{2} \leq \arg \beta(y) \leq \pi
$$

for almost all $y \in \Gamma$. Clearly (18) holds if $\operatorname{Re}(\beta) \geq 0$, the case of prime practical interest, since $\operatorname{Re}(\beta) \geq 0$ if and only if the boundary $\Gamma$ does not emit acoustic energy. We then define the angle $\Phi \in[-\pi / 2,0]$, by

$$
\Phi:=\min \left\{0, \text { ess } \inf _{y \in \Gamma} \arg \beta(y)\right\} .
$$


In order to show the boundary value problem is well-posed we will make some assumptions on $\beta \in L^{\infty}(\Gamma)$. In the $2 \mathrm{D}$ case, when $\Gamma$ is a straight line and $\beta$ is a constant, it is well-known that the boundary value problem is ill-posed if $\beta=-i s$, for some $s>0$. This motivates the following condition, that

$$
\operatorname{dist}[\beta(\Gamma),\{-i s: s \geq 0\}]>0,
$$

where $\beta(\Gamma)$ is the essential range of $\beta$.

Our main results will require that one or other of the following assumptions on $\beta \in L^{\infty}(\Gamma)$ hold.

Assumption 1 (A1). For some $\alpha_{1} \in[0, \pi / 2), \eta>0$, it holds that

$$
\operatorname{Im}\left[e^{i \alpha_{1}} \beta\right] \geq \eta \quad \text { for almost all } y \in \Gamma \text {. }
$$

When we make use of (A1) it will be convenient to employ the notation $\eta_{\alpha}=\eta \sec \alpha_{1}$.

Assumption 2 (A2). For some $\eta>0$,

$$
\operatorname{Re}(\beta) \geq \eta \quad \text { for almost all } y \in \Gamma
$$

Note that both these assumptions imply that $\beta$ satisfies both (18) and (20).

Remark 3.1. Our analysis of the variational problem under assumption (A1) will not be applicable to the limiting case when $\alpha_{1}=\pi / 2$, so a different study of the variational problem will be made under (A2). Note that if $\beta$ satisfies (A2), then it satisfies (A1), but results with less restriction on $\kappa$ are obtained under (A2). Moreover, a series of functions $\beta_{n}$ satisfying (A2) for some $\eta>0$, will satisfy (A1) for some values $\eta_{\alpha_{n}}>0$. However these values $\eta_{\alpha_{n}}$ could become arbitrarily small. It thus makes sense to study the problem separately under (A2).

Our main theorem of this section is then the following:

Theorem 4. i) Suppose (A1) holds and that

$$
\kappa<\frac{2 \eta_{\alpha}}{1+\sqrt{1+2 \eta_{\alpha}^{2}}}
$$

Then for some constant $C_{1}>0$ such that

$$
C_{1} \leq \sec \alpha_{1}\left[\frac{2 \eta_{\alpha}+\eta_{\alpha} \kappa^{2}+4 \kappa+\sqrt{\left[\eta_{\alpha}\left(2+\kappa^{2}\right)-4 \kappa\right]^{2}+16 \kappa^{3} \eta_{\alpha}}}{6 \eta_{\alpha}-\eta_{\alpha} \kappa^{2}-4 \kappa-\sqrt{\left[\eta_{\alpha}\left(2+\kappa^{2}\right)-4 \kappa\right]^{2}+16 \kappa^{3} \eta_{\alpha}}}\right]
$$

it holds that

$$
|c(u, u)| \geq C_{1}^{-1}\|u\|_{H^{1}\left(S_{H}\right)}^{2}, \quad u \in H^{1}\left(S_{H}\right),
$$

so that the variational problem (17) is uniquely solvable, and the solution satisfies the estimate

$$
\|u\|_{H^{1}\left(S_{H}\right)} \leq C_{1}\|\mathcal{G}\|_{H^{1}\left(S_{H}\right)^{*}}
$$

In particular, the scattering problem (16) is uniquely solvable and the solution satisfies the bound

$$
k\|u\|_{H^{1}\left(S_{H}\right)} \leq C_{1}\|g\|_{2} .
$$

ii) Suppose (A2) holds and that $\kappa<\sqrt{2}$. Then for some constant $C_{2}>0$ such that

$$
C_{2} \leq \frac{6+\kappa^{2}}{2-\kappa^{2}}\left[1+\frac{1}{\eta^{2}}\left(\eta \tan (-\Phi)+\frac{8 \kappa}{6+\kappa^{2}} \frac{\left(2+\kappa^{2}\right)}{\left(2-\kappa^{2}\right)}\right)^{2}\right]^{\frac{1}{2}}
$$


it holds that

$$
|c(u, u)| \geq C_{2}^{-1}\|u\|_{H^{1}\left(S_{H}\right)}^{2}, \quad u \in H^{1}\left(S_{H}\right),
$$

so that the variational problem (17) is uniquely solvable, and the solution satisfies the estimate

$$
\|u\|_{H^{1}\left(S_{H}\right)} \leq C_{2}\|\mathcal{G}\|_{H^{1}\left(S_{H}\right)^{*}}
$$

In particular, the scattering problem (16) is uniquely solvable, and the solution satisfies the bound

$$
k\|u\|_{H^{1}\left(S_{H}\right)} \leq C_{2}\|g\|_{2} .
$$

Theorem 4 will be proved via a sequence of lemmas. Our first aim is to show that the sesquilinear form $c$ is bounded. For this we will need the following trace results which are proved by combining standard methods of proof used for trace theorems on bounded domains, together with the proof of [21, Lemma 3.4]. The proof can be found in the appendix of [4].

Lemma 5. Let $D$ be an $(L, \mu, N)$ Lipschitz domain, and let $S_{H}=D \backslash \overline{U_{H}}$ for $H \geq f_{+}+\mu$. For $u \in H^{1}\left(S_{H}\right)$,

$$
\left\|\gamma_{-} u\right\|_{H^{\frac{1}{2}\left(\Gamma_{H}\right)}} \leq \sqrt{\left(1+\frac{1}{k \mu}\right)}\|u\|_{H^{1}\left(S_{H}\right)}
$$

and the map $\gamma_{*}: \mathcal{D}\left(S_{H}\right) \rightarrow L^{2}(\Gamma)$ such that $\gamma_{*} u$ is $u$ restricted to $\Gamma$, for $u \in \mathcal{D}\left(S_{H}\right)$, extends to a bounded linear operator $\gamma_{*}: H^{1}\left(S_{H}\right) \rightarrow L^{2}(\Gamma)$ with

$$
k\left\|\gamma_{*} u\right\|_{L^{2}(\Gamma)}^{2} \leq N L^{\prime}\left(1+\frac{1}{k \mu}\right)\|u\|_{H^{1}\left(S_{H}\right)}^{2} .
$$

Let $B:=\|\beta\|_{L^{\infty}(\Gamma)}$. Let us now show that the sesquilinear form $c(.,$.$) is bounded.$

Lemma 6. Let $D$ be an $(L, \mu, N)$ Lipschitz domain and let $S_{H}=D \backslash \overline{U_{H}}$ for $H \geq f_{+}+\mu$. For all $u, v \in H^{1}\left(S_{H}\right)$,

$$
|c(u, v)| \leq\left(1+\left(1+B N L^{\prime}\right)\left[1+\frac{1}{k \mu}\right]\right)\|u\|_{H^{1}\left(S_{H}\right)}\|v\|_{H^{1}\left(S_{H}\right)} .
$$

Proof. This follows from the definition of $c(.,$.$) , the Cauchy-Schwarz inequality,$ Lemma 5 and the mapping properties of $T$.

We now prove an important Friedrich's type inequality.

Lemma 7. Let $S_{H}$ be an $(L, \mu, N+1)$ Lipschitz domain. Then for all $w \in$ $H^{1}\left(S_{H}\right)$ and for all $\zeta>0$

$$
\|w\|_{2}^{2} \leq(1+\zeta) \frac{\left(H-f_{-}\right)^{2}}{2}\left\|\frac{\partial w}{\partial x_{n}}\right\|_{2}^{2}+\left(1+\frac{1}{\zeta}\right)\left(H-f_{-}\right)\|w\|_{L^{2}(\Gamma)}^{2} .
$$

Proof. For $x=\left(\tilde{x}, x_{n}\right) \in S_{H}$, define $x_{B}: S_{H} \rightarrow \mathbf{R}$ by $x_{B}=\max \{t: t \leq$ $x_{n}$ and $\left.(\tilde{x}, t) \in \Gamma\right\}$. Note that because $\Gamma$ possesses the strong local Lipschitz property, $x_{B}$ is Borel measurable. 
Now for $w \in \mathcal{D}\left(S_{H}\right)$,

$$
\begin{aligned}
|w(x)|^{2}= & \left|\int_{x_{B}}^{x_{n}} \frac{\partial w\left(\tilde{x}, y_{n}\right)}{\partial y_{n}} d y_{n}+w\left(\tilde{x}, x_{B}\right)\right|^{2} \\
\leq & \left(\int_{x_{B}}^{x_{n}}\left|\frac{\partial w\left(\tilde{x}, y_{n}\right)}{\partial y_{n}}\right| d y_{n}+\left|w\left(\tilde{x}, x_{B}\right)\right|\right)^{2} \\
\leq & \left(x_{n}-x_{B}\right) \int_{x_{B}}^{x_{n}}\left|\frac{\partial w\left(\tilde{x}, y_{n}\right)}{\partial y_{n}}\right|^{2} d y_{n} \\
& +2\left|w\left(\tilde{x}, x_{B}\right)\right| \int_{x_{B}}^{x_{n}}\left|\frac{\partial w\left(\tilde{x}, y_{n}\right)}{\partial y_{n}}\right| d y_{n}+\left|w\left(\tilde{x}, x_{B}\right)\right|^{2} \\
\leq & (1+\zeta)\left(x_{n}-x_{B}\right) \int_{x_{B}}^{x_{n}}\left|\frac{\partial w\left(\tilde{x}, y_{n}\right)}{\partial y_{n}}\right|^{2} d y_{n}+\left(1+\frac{1}{\zeta}\right)\left|w\left(\tilde{x}, x_{B}\right)\right|^{2} \\
\leq & (1+\zeta)\left(x_{n}-f_{-}\right) \int_{\mathbf{R}} 1_{S_{H}}\left(\tilde{x}, y_{n}\right)\left|\frac{\partial w\left(\tilde{x}, y_{n}\right)}{\partial y_{n}}\right|^{2} d y_{n}+\left(1+\frac{1}{\zeta}\right)\left|w\left(\tilde{x}, x_{B}\right)\right|^{2},
\end{aligned}
$$

so that, since $\int_{\mathbf{R}} 1_{S_{H}}\left(x_{n}-f_{-}\right) d x_{n} \leq\left(H-f_{-}\right)^{2} / 2$, we have, using Fubini's Theorem,

$$
\begin{aligned}
& \int_{S_{H}}|w(x)|^{2} d x \\
& \leq(1+\zeta) \int_{\mathbf{R}^{n-1}} \int_{\mathbf{R}} 1_{S_{H}}\left(\tilde{x}, x_{n}\right)\left(x_{n}-f_{-}\right) d x_{n}\left(\int_{\mathbf{R}} 1_{S_{H}}\left(\tilde{x}, y_{n}\right)\left|\frac{\partial w\left(\tilde{x}, y_{n}\right)}{\partial y_{n}}\right|^{2} d y_{n}\right) d \tilde{x} \\
& \quad+\left(1+\frac{1}{\zeta}\right) \int_{\mathbf{R}} \int_{\mathbf{R}^{n-1}} 1_{S_{H}}\left(\tilde{x}, x_{n}\right)\left|w\left(\tilde{x}, x_{B}\right)\right|^{2} d \tilde{x} d x_{n} \\
& \leq(1+\zeta) \frac{\left(H-f_{-}\right)^{2}}{2} \int_{\mathbf{R}^{n-1}} \int_{\mathbf{R}} 1_{S_{H}}\left(\tilde{x}, y_{n}\right)\left|\frac{\partial w\left(\tilde{x}, y_{n}\right)}{\partial y_{n}}\right|^{2} d y_{n} d \tilde{x} \\
& \quad+\left(1+\frac{1}{\zeta}\right)\left(H-f_{-}\right) \int_{\Gamma}|w(s)|^{2} d s,
\end{aligned}
$$

and the result follows for $w \in \mathcal{D}\left(S_{H}\right)$. By the density of this space in $H^{1}\left(S_{H}\right)$, the result holds for all $w \in H^{1}\left(S_{H}\right)$.

We now recall Lemma 3.2 from [21].

Lemma 8. For all $\phi, \psi \in H^{1 / 2}\left(\Gamma_{H}\right)$,

$$
\int_{\Gamma_{H}} \phi T \psi d s=\int_{\Gamma_{H}} \psi T \phi d s
$$

For all $\phi \in H^{1 / 2}\left(\Gamma_{H}\right)$,

$$
\operatorname{Re} \int_{\Gamma_{H}} \bar{\phi} T \phi d s \geq 0, \quad \operatorname{Im} \int_{\Gamma_{H}} \bar{\phi} T \phi d s \leq 0 .
$$

Employing this lemma it is easy to verify the following important symmetry property of $c$ : for all $u, v \in H^{1}\left(S_{H}\right)$

$$
c(v, u)=c(\bar{u}, \bar{v}) .
$$

We now introduce $\alpha_{2} \in(-\Phi, \pi / 2]$ such that

$$
\tan \alpha_{2}=\frac{1}{\eta}\left[\eta \tan (-\Phi)+\frac{8 \kappa}{6+\kappa^{2}} \frac{\left(2+\kappa^{2}\right)}{\left(2-\kappa^{2}\right)}\right] .
$$


We then define the sesquilinear forms $c_{1}, c_{2}: H^{1}\left(S_{H}\right) \times H^{1}\left(S_{H}\right) \rightarrow \mathbf{C}$ via

$$
c_{1}(u, v)=e^{i \alpha_{1}} c(u, v), \quad c_{2}(u, v)=e^{i \alpha_{2}} c(u, v), \quad u, v \in H^{1}\left(S_{H}\right) .
$$

(Note that the definition of $\alpha_{2}$ was motivated in trying to show ellipticity of $c_{2}$ and recall that $\alpha_{1}$ was defined when we wrote down (A1).) We now show that the sesquilinear forms $c_{1}, c_{2}$ are elliptic for small $\kappa$.

Lemma 9. i) If (A1) holds, then for all $w \in H^{1}\left(S_{H}\right)$

$$
\operatorname{Re}\left[c_{1}(w, w)\right] \geq C\|w\|_{H^{1}\left(S_{H}\right)},
$$

where

$$
C=\cos \alpha_{1}\left(\frac{6 \eta_{\alpha}-\eta_{\alpha} \kappa^{2}-4 \kappa-\sqrt{\left[\eta_{\alpha}\left(2+\kappa^{2}\right)-4 \kappa\right]^{2}+16 \kappa^{3} \eta_{\alpha}}}{2 \eta_{\alpha}+\eta_{\alpha} \kappa^{2}+4 \kappa+\sqrt{\left[\eta_{\alpha}\left(2+\kappa^{2}\right)-4 \kappa\right]^{2}+16 \kappa^{3} \eta_{\alpha}}}\right) .
$$

ii) If (A2) holds, then for all $w \in H^{1}\left(S_{H}\right)$

$$
\operatorname{Re}\left[c_{2}(w, w)\right] \geq C\|w\|_{H^{1}\left(S_{H}\right)},
$$

where

$$
C=\frac{2-\kappa^{2}}{6+\kappa^{2}}\left[1+\frac{1}{\eta^{2}}\left(\eta \tan (-\Phi)+\frac{8 \kappa}{6+\kappa^{2}} \frac{\left(2+\kappa^{2}\right)}{\left(2-\kappa^{2}\right)}\right)^{2}\right]^{-\frac{1}{2}}
$$

Proof. i) For $w \in H^{1}\left(S_{H}\right)$

$$
\begin{aligned}
\operatorname{Re}\left[c_{1}(w, w)\right]= & \cos \alpha_{1}\left[\|\nabla w\|_{2}^{2}-k^{2}\|w\|_{2}^{2}\right]+\operatorname{Re}\left[e^{i \alpha_{1}} \int_{\Gamma_{H}} \gamma_{-} \bar{w} T \gamma_{-} w d s\right] \\
& +k \int_{\Gamma} \operatorname{Im}\left[e^{i \alpha_{1}} \beta\right]|w|^{2} d s .
\end{aligned}
$$

By Lemma 8, $\arg \left\{\int_{\Gamma_{H}} \gamma_{-} \bar{w} T \gamma_{-} w d s\right\} \in[-\pi / 2,0]$, so that

$$
\operatorname{Re}\left[e^{i \alpha_{1}} \int_{\Gamma_{H}} \gamma_{-} \bar{w} T \gamma_{-} w d s\right] \geq 0
$$

because $\alpha_{1} \in[0, \pi / 2)$. Hence, using Lemma 7 with $\zeta>0$, noting $\operatorname{Im}\left[e^{i \alpha_{1}} \beta\right] \geq \eta$, and, where $\theta>0$, we have

$$
\begin{aligned}
\operatorname{Re}\left[c_{1}(w, w)\right] \geq & \cos \alpha_{1}\left(1-(1+\zeta) \frac{\kappa^{2}}{2}(1+\theta)\right)\|\nabla w\|_{2}^{2} \\
& +k\left(\eta-\cos \alpha_{1}\left(1+\zeta^{-1}\right)(1+\theta) \kappa\right) \int_{\Gamma}|w|^{2} d s+\cos \alpha_{1} \theta k^{2}\|w\|_{2}^{2} .
\end{aligned}
$$

To obtain from this estimate the optimal ellipticity bound we need to make

$$
\eta-\cos \alpha_{1}\left(1+\zeta^{-1}\right)(1+\theta) \kappa=0 \quad \text { and } \quad \theta=1-(1+\zeta) \frac{\kappa^{2}}{2}(1+\theta) .
$$

This is achieved by setting

$$
\theta=\frac{2-\kappa^{2}[1+\zeta]}{2+\kappa^{2}[1+\zeta]}
$$

and

$$
\zeta=\frac{1}{2 \eta_{\alpha} \kappa^{2}}\left(-\left[\eta_{\alpha}\left(2+\kappa^{2}\right)-4 \kappa\right]+\sqrt{\left[\eta_{\alpha}\left(2+\kappa^{2}\right)-4 \kappa\right]^{2}+16 \kappa^{3} \eta_{\alpha}}\right)
$$


ii) As in part i) we have, for $w \in H^{1}\left(S_{H}\right)$, and $\theta>0, \zeta>0$

$$
\begin{aligned}
\operatorname{Re}\left[c_{2}(w, w)\right] \geq & \cos \alpha_{2}\left(1-(1+\zeta) \frac{\kappa^{2}}{2}(1+\theta)\right)\|\nabla w\|_{2}^{2} \\
& -k \cos \alpha_{2}\left(1+\zeta^{-1}\right)(1+\theta) \kappa \int_{\Gamma}|w|^{2} d s \\
& +k \int_{\Gamma} \operatorname{Im}\left[e^{i \alpha_{2}} \beta\right]|w|^{2} d s+\cos \alpha_{2} \theta k^{2}\|w\|_{2}^{2}
\end{aligned}
$$

Now, if $\eta\left(\alpha_{2}\right):=\operatorname{ess~inf}_{y \in \Gamma} \operatorname{Im}\left[e^{i \alpha_{2}} \beta\right]$, it is evident, since $\alpha_{2}>-\Phi$, that

$$
\eta\left(\alpha_{2}\right) \geq \operatorname{Im}\left[e^{i\left(\alpha_{2}+\Phi\right)} \frac{\eta}{\cos \Phi}\right]=\frac{\eta \sin \left(\alpha_{2}+\Phi\right)}{\cos \Phi}=\eta\left[\sin \alpha_{2}+\cos \alpha_{2} \tan \Phi\right] .
$$

So making the (optimal) choices

$$
\zeta=\frac{1}{\kappa^{2}}-\frac{1}{2} \quad \text { and } \quad \theta=\frac{2-\kappa^{2}}{6+\kappa^{2}}
$$

(28) becomes

$$
\begin{aligned}
\operatorname{Re}\left[c_{2}(w, w)\right] \geq & \cos \alpha_{2}\left[\frac{2-\kappa^{2}}{6+\kappa^{2}}\right]\|w\|_{H^{1}\left(S_{H}\right)} \\
& +k\left[\eta\left(\sin \alpha_{2}+\cos \alpha_{2} \tan \Phi\right)-\cos \alpha_{2} \frac{\left(2+\kappa^{2}\right)}{\left(2-\kappa^{2}\right)}\left(\frac{8 \kappa}{6+\kappa^{2}}\right)\right] \int_{\Gamma}|w|^{2} d s,
\end{aligned}
$$

so that the desired bound holds because (27) implies that

$$
\left[\eta\left(\sin \alpha_{2}+\cos \alpha_{2} \tan \Phi\right)-\cos \alpha_{2} \frac{\left(2+\kappa^{2}\right)}{\left(2-\kappa^{2}\right)}\left(\frac{8 \kappa}{6+\kappa^{2}}\right)\right]=0,
$$

and because

$$
\cos \alpha_{2}=\frac{1}{\sqrt{1+\tan ^{2} \alpha_{2}}} .
$$

Using Lemmas 6 and 9, we can now prove Theorem 4.

Proof. By Lemma 9 and under the assumption that $\kappa<2 \eta_{\alpha} /\left(1+\sqrt{1+2 \eta_{\alpha}^{2}}\right)$ (respectively $\kappa<\sqrt{2}$ ), one can verify that $c_{1}$, (resp. $c_{2}$ ) is elliptic. Lemma 6 implies that $c_{1}$ (resp. $c_{2}$ ) is bounded and hence by the Lax-Milgram lemma the existence of a unique solution $u$ to

$$
c_{1}(u, v)=\mathcal{G}(v) \quad\left(\text { resp. } \quad c_{2}(u, v)=\mathcal{G}(v)\right)
$$

for a given $\mathcal{G} \in H^{1}\left(S_{H}\right)^{*}$ and for all $v \in H^{1}\left(S_{H}\right)$ is assured, assuming (A1), (resp. (A2)). It is then trivial to show that for a given $\mathcal{G} \in H^{1}\left(S_{H}\right)^{*}$ there exists a unique solution to the problem

$$
c(u, v)=\mathcal{G}(v), \quad v \in H^{1}\left(S_{H}\right) .
$$

Moreover, the estimates (22), (24) also follow from the Lax-Milgram lemma and the simple relationship between $c_{1}$ (resp. $\left.c_{2}\right)$ and $c$. In the particular case $\mathcal{G}(v)=-(g, v)$, for some $g \in L^{2}\left(S_{H}\right)$ we have

$$
\|\mathcal{G}\|_{H^{1}\left(S_{H}\right)^{*}}=\sup _{\phi \in H^{1}\left(S_{H}\right)} \frac{|(g, \phi)|}{\|\phi\|_{H^{1}\left(S_{H}\right)}} \leq\|g\|_{2} \sup _{\phi \in H^{1}\left(S_{H}\right)} \frac{\|\phi\|_{2}}{\|\phi\|_{H^{1}\left(S_{H}\right)}} \leq \frac{1}{k}\|g\|_{2},
$$

so that (23) and (25) hold. 


\section{Analysis of the variational problem at arbitrary frequency}

The sesquilinear form $c$ is not elliptic if the wavenumber $k$ is large. In this section, we will assume that $\Gamma$ is the graph of a Lipschitz function. Under this restriction we will employ Babuška's generalised Lax-Milgram theorem to show that the boundary value problem is well-posed.

Our main result is obtained for $\beta \in L^{\infty}(\Gamma)$ satisfying assumption (A2), i.e. that $\operatorname{Re}(\beta) \geq \eta$ for some $\eta>0$, for almost all $y \in \Gamma$. This implies that

$$
\Phi \in\left(-\frac{\pi}{2}, 0\right]
$$

where $\Phi$ is given by (19). Note that

$$
\sec \Phi \leq \frac{\sqrt{\eta^{2}+B^{2}}}{\eta}
$$

where $B=\|\beta\|_{L^{\infty}(\Gamma)}$. Clearly $\sec \Phi=1$ if $0 \leq \arg \beta(y)<\frac{\pi}{2}$ for almost all $y \in \Gamma$.

Theorem 10. If $\Gamma$ is given by (3) with $f$ satisfying (1), and (A2) holds then the variational problem (17) has a unique solution $u \in H^{1}\left(S_{H}\right)$ for every $\mathcal{G} \in H^{1}\left(S_{H}\right)^{*}$ and

$$
\|u\|_{H^{1}\left(S_{H}\right)} \leq \sec \Phi(1+2 E)\|\mathcal{G}\|_{H^{1}\left(S_{H}\right)^{*}}
$$

where

$$
E=\left(2 \sqrt{2} \kappa\left[\frac{2+\kappa^{2}\left(1+B^{2}(1+L)\right)}{\eta}+\kappa[\sqrt{2}+\sec \Phi]\right]+\frac{\sec \Phi}{4 \sqrt{2}}\right) .
$$

In particular, the boundary value problem and the equivalent variational problem have exactly one solution, and the solution satisfies the bound

$$
k\|u\|_{H^{1}\left(S_{H}\right)} \leq E\|g\|_{2} .
$$

To apply the generalised Lax-Milgram theorem we need to show that $c$ is bounded, which we have done in Lemma 6; to establish the inf-sup condition that

$$
\chi:=\inf _{0 \neq u \in H^{1}\left(S_{H}\right)} \sup _{0 \neq v \in H^{1}\left(S_{H}\right)} \frac{|c(u, v)|}{\|u\|_{H^{1}\left(S_{H}\right)}\|v\|_{H^{1}\left(S_{H}\right)}}>0 ;
$$

and to establish a "transposed" inf-sup condition. It follows easily from (26) that a transposed inf-sup condition can be automatically deduced if (32) holds, i.e. that the following lemma (cf. [21, Lemma 4.2]) is true.

Lemma 11. If (32) holds, then for all non-zero $v \in H^{1}\left(S_{H}\right)$,

$$
\sup _{0 \neq u \in H^{1}\left(S_{H}\right)} \frac{|c(u, v)|}{\|u\|_{H^{1}\left(S_{H}\right)}}>0 .
$$

The following result follows from [36, Theorem 2.15] and Lemmas 6 and 11.

Corollary 12. If (32) holds, then the variational problem (17) has exactly one solution $u \in H^{1}\left(S_{H}\right)$ for all $\mathcal{G} \in H^{1}\left(S_{H}\right)^{*}$. Moreover,

$$
\|u\|_{H^{1}\left(S_{H}\right)} \leq \chi^{-1}\|\mathcal{G}\|_{H^{1}\left(S_{H}\right)^{*}} .
$$

To show (32) we will establish an apriori bound for solutions of (17), from which the inf-sup condition will follow by the following easily established lemma (see [36, Remark 2.20]). 
Lemma 13. Suppose that there exists $C>0$ such that, for all $u \in H^{1}\left(S_{H}\right)$ and $\mathcal{G} \in H^{1}\left(S_{H}\right)^{*}$ satisfying (17) it holds that

$$
\|u\|_{H^{1}\left(S_{H}\right)} \leq C\|\mathcal{G}\|_{H^{1}\left(S_{H}\right)^{*}} .
$$

Then the inf-sup condition (32) holds with $\chi \geq C^{-1}$.

The following lemma reduces the problem of establishing (33) to that of establishing an a priori bound for solutions of the special case (16).

Lemma 14. Suppose there exists $C^{*}>0$ such that, for all $u \in H^{1}\left(S_{H}\right)$ and $g \in L^{2}\left(S_{H}\right)$ satisfying (16) it holds that

$$
\|u\|_{H^{1}\left(S_{H}\right)} \leq k^{-1} C^{*}\|g\|_{2} .
$$

Then, for all $u \in H^{1}\left(S_{H}\right)$ and $\mathcal{G} \in H^{1}\left(S_{H}\right)^{*}$ satisfying (17), the bound (33) holds with

$$
C \leq \sec \Phi\left(1+2 C^{*}\right) .
$$

Proof. Let $\hat{c}: H^{1}\left(S_{H}\right) \times H^{1}\left(S_{H}\right) \rightarrow \mathbf{C}$ be defined by

$$
\begin{aligned}
\hat{c}(u, v): & =e^{-i \Phi}\left[c(u, v)+2 k^{2}(u, v)\right] \\
& =e^{-i \Phi}\left[(\nabla u, \nabla v)+k^{2}(u, v)+\int_{\Gamma_{H}} \gamma_{-} \bar{v} T \gamma_{-} u d s-\int_{\Gamma} i k \beta \gamma_{*} u \gamma_{*} \bar{v} d s\right],
\end{aligned}
$$

for $u, v \in H^{1}\left(S_{H}\right)$. For $u \in H^{1}\left(S_{H}\right)$ we see that

$$
\operatorname{Re}[\hat{c}(u, u)] \geq \operatorname{Re}\left(e^{-i \Phi}\|u\|_{H^{1}\left(S_{H}\right)}^{2}\right)=\cos \Phi\|u\|_{H^{1}\left(S_{H}\right)}^{2} .
$$

This follows because by Lemma $8, \arg \left\{\int_{\Gamma_{H}} \gamma_{-} \bar{u} T \gamma_{-} u d s\right\} \in[-\pi / 2,0]$, whilst $\Phi \in$ $(-\pi / 2,0]$, so that, noting the definition of $\Phi$, it holds that

$$
\operatorname{Re}\left(e^{-i \Phi} \int_{\Gamma_{H}} \gamma_{-} \bar{u} T \gamma_{-} u d s\right) \geq 0, \quad \operatorname{Re}\left(-e^{-i \Phi} \int_{\Gamma} i k \beta|u|^{2} d s\right) \geq 0 .
$$

Thus given $\mathcal{G} \in H^{1}\left(S_{H}\right)^{*}$, it follows, by the Lax-Milgram lemma, that there exists unique $u_{0} \in H^{1}\left(S_{H}\right)$ satisfying

$$
\hat{c}\left(u_{0}, v\right)=\mathcal{G}(v), \quad v \in H^{1}\left(S_{H}\right),
$$

and moreover $u_{0}$ satisfies the estimate

$$
\left\|u_{0}\right\|_{H^{1}\left(S_{H}\right)} \leq \sec \Phi\|\mathcal{G}\|_{H^{1}\left(S_{H}\right)^{*}} .
$$

Now suppose $u \in H^{1}\left(S_{H}\right)$ and $\mathcal{G} \in H^{1}\left(S_{H}\right)^{*}$ satisfy

$$
c(u, v)=\mathcal{G}(v), \quad v \in H^{1}\left(S_{H}\right),
$$

and denote by $u_{0} \in H^{1}\left(S_{H}\right)$ the unique solution of (35). Then, defining $w=u-$ $e^{-i \Phi} u_{0}$, we see that

$$
\begin{aligned}
c(w, v) & =c(u, v)-\hat{c}\left(u_{0}, v\right)+e^{-i \Phi} 2 k^{2}\left(u_{0}, v\right) \\
& =\mathcal{G}(v)-\mathcal{G}(v)+e^{-i \Phi} 2 k^{2}\left(u_{0}, v\right)=e^{-i \Phi} 2 k^{2}\left(u_{0}, v\right),
\end{aligned}
$$

for all $v \in H^{1}\left(S_{H}\right)$. Thus $w$ satisfies (16) with $g=-e^{-i \Phi} 2 k^{2} u_{0}$. It follows, using (34) and (36), that

$$
\|w\|_{H^{1}\left(S_{H}\right)} \leq k^{-1} C^{*}\left\|2 k^{2} u_{0}\right\|_{2} \leq 2 C^{*} \sec \Phi\|\mathcal{G}\|_{H^{1}\left(S_{H}\right)^{*}}
$$

The bound (33), with $C \leq \sec \Phi\left(1+2 C^{*}\right)$, follows from (36) and (38). 
We now turn to establishing the a priori bound (34), at first just for the case when $\Gamma$ is the graph of a smooth Lipschitz function and $\beta \in C^{\infty}(\Gamma) \cap L^{\infty}(\Gamma)$.

Remark 4.1. If $v \in H^{1}\left(S_{H}\right)$, then $\gamma_{*} v \in L^{2}(\Gamma)$ by Lemma 5. For Lemma 15 it will be necessary to know that $\gamma_{*} v \in H_{\text {loc }}^{\frac{1}{2}}(\Gamma)$, as defined in [44]. This follows from [44, Theorem 3.37].

We recall that $\nu$ is the outward unit normal to $S_{H}$ and $\nu_{n}=\nu \cdot e_{n}$ is the $n$th (vertical) component of $\nu$.

Lemma 15. Suppose $\Gamma$ is given by (3) with $f$ satisfying (2) and with $f \in$ $C^{\infty}\left(\mathbf{R}^{n-1}\right)$. Let $H \geq f_{+}+\mu, g \in L^{2}\left(S_{H}\right)$ and let $\beta \in C^{\infty}(\Gamma) \cap L^{\infty}(\Gamma)$ be such that (A2) holds. Suppose $w \in H^{1}\left(S_{H}\right)$ satisfies

$$
b(w, \phi)=-(g, \phi), \quad \phi \in H^{1}\left(S_{H}\right) .
$$

Then

$$
k\|w\|_{H^{1}\left(S_{H}\right)} \leq\left(2 \sqrt{2} \kappa\left[\frac{2+\kappa^{2}\left(1+B^{2}(1+L)\right)}{\eta}+\kappa[\sqrt{2}+\sec \Phi]\right]+\frac{\sec \Phi}{4 \sqrt{2}}\right)\|g\|_{2} .
$$

Proof. Setting $\phi=w$ in (39) and, multiplying through by $e^{-i \Phi}$, and taking real parts (c.f. the proof of Lemma 14) we derive the estimate

$$
\|\nabla w\|_{2}^{2} \leq k^{2}\|w\|_{2}^{2}+\sec \Phi\|g\|_{2}\|w\|_{2}
$$

Setting $\phi=w$ in (39) and taking imaginary parts, and writing $\gamma_{*} w$ as $w$, gives

$$
\operatorname{Im} \int_{\Gamma_{H}} \gamma_{-} \bar{w} T \gamma_{-} w d s-\int_{\Gamma} k \operatorname{Re}(\beta)|w|^{2} d s=-\operatorname{Im}(g, w),
$$

so that from Lemma 8 and assuming (A2) we get

$$
\eta \int_{\Gamma} k|w|^{2} d s \leq\|g\|_{2}\|w\|_{2}
$$

From Lemma 7 with $\zeta=1$, we have

$$
k^{2}\|w\|_{2}^{2} \leq \kappa^{2}\left\|\frac{\partial w}{\partial x_{n}}\right\|_{2}^{2}+2 k \kappa \int_{\Gamma}|w|^{2} d s
$$

Extending the definition of $w$ to $D$ by defining $w$ in $U_{H}$ by (9) with $F_{H}:=\gamma_{-} w$, it follows from Theorem 3 that $w$ satisfies the boundary value problem, with $g$ extended by zero from $S_{H}$ to $D$.

With $\beta \in C^{\infty}(\Gamma) \cap L^{\infty}(\Gamma)$ and $\left.w\right|_{\Gamma} \in H_{\text {loc }}^{\frac{1}{2}}(\Gamma)$ it follows (e.g. [44, Theorem 3.20]) that $\beta w \in H_{\mathrm{loc}}^{\frac{1}{2}}(\Gamma)$. Together with the assumptions that $g \in L^{2}(D)$, and that the boundary is smooth, regularity theory implies that $w \in H_{\text {loc }}^{2}(D)$ (e.g. [44, Theorem 4.18]).

Let $r=|\tilde{x}|$. For $A \geq 1$ let $\phi_{A} \in C_{0}^{\infty}(\mathbf{R})$ be such that $0 \leq \phi_{A} \leq 1, \phi_{A}(r)=1$ if $r \leq A$ and $\phi_{A}(r)=0$ if $r \geq A+1$ and finally such that $\left\|\phi_{A}^{\prime}\right\|_{\infty} \leq M$ for some fixed $M$ independent of $A$. 
In view of this regularity and since $w$ satisfies the boundary value problem, we have

$$
\begin{aligned}
& 2 \operatorname{Re} \int_{S_{H}} \phi_{A}(r)\left(x_{n}-H\right) g \frac{\partial \bar{w}}{\partial x_{n}} d x=2 \operatorname{Re} \int_{S_{H}} \phi_{A}(r)\left(x_{n}-H\right)\left(\Delta w+k^{2} w\right) \frac{\partial \bar{w}}{\partial x_{n}} d x \\
& =\int_{S_{H}}\left\{2 \operatorname{Re}\left\{\nabla \cdot\left(\phi_{A}(r)\left(x_{n}-H\right) \frac{\partial \bar{w}}{\partial x_{n}} \nabla w\right)\right\}-2 \phi_{A}(r)\left|\frac{\partial w}{\partial x_{n}}\right|^{2}\right. \\
& \quad-2 \operatorname{Re}\left[\left(x_{n}-H\right) \phi_{A}(r) \frac{\partial \nabla \bar{w}}{\partial x_{n}} \cdot \nabla w\right]-2 \phi_{A}^{\prime}(r)\left(x_{n}-H\right) \frac{\tilde{x}}{|\tilde{x}|} \cdot \operatorname{Re}\left(\nabla_{\tilde{x}} w \frac{\partial \bar{w}}{\partial x_{n}}\right) \\
& \left.\quad+2 \operatorname{Re}\left[k^{2}\left(x_{n}-H\right) \phi_{A}(r) \frac{\partial \bar{w}}{\partial x_{n}} w\right]\right\} d x .
\end{aligned}
$$

Using the divergence theorem and integration by parts

$$
\begin{aligned}
& 2 \operatorname{Re} \int_{S_{H}} \phi_{A}(r)\left(x_{n}-H\right) g \frac{\partial \bar{w}}{\partial x_{n}} d x \\
& =-\int_{\Gamma}\left(x_{n}-H\right) \phi_{A}(r)\left\{\nu_{n}\left(|\nabla w|^{2}-k^{2}|w|^{2}\right)-2 \operatorname{Re}\left(\frac{\partial \bar{w}}{\partial x_{n}} \frac{\partial w}{\partial \nu}\right)\right\} d s \\
& \quad+\int_{S_{H}}\left\{\phi_{A}(r)\left(|\nabla w|^{2}-k^{2}|w|^{2}-2\left|\frac{\partial w}{\partial x_{n}}\right|^{2}\right)-2 \phi_{A}^{\prime}(r)\left(x_{n}-H\right) \operatorname{Re}\left(\frac{\partial \bar{w}}{\partial x_{n}} \frac{\partial w}{\partial r}\right)\right\} d x .
\end{aligned}
$$

Now, on $\Gamma \cap \operatorname{supp}\left(\phi_{A}(r)\right)$

$$
\frac{\partial w}{\partial x_{n}}=e_{n} \cdot \nabla w=e_{n} \cdot\left(\nabla_{\Gamma} w+\frac{\partial w}{\partial \nu} \nu\right)
$$

where $\nabla_{\Gamma} w$, the tangential part of $\nabla w$, is given by

$$
\nabla_{\Gamma} w:=\nabla w-\frac{\partial w}{\partial \nu} \nu
$$

So

$$
\operatorname{Re}\left(\frac{\partial \bar{w}}{\partial x_{n}} \frac{\partial w}{\partial \nu}\right)=\left|\frac{\partial w}{\partial \nu}\right|^{2} \nu_{n}+\operatorname{Re}\left(\left(e_{n} \cdot \nabla_{\Gamma} \bar{w}\right) \frac{\partial w}{\partial \nu}\right)
$$

Also

$$
|\nabla w|^{2}=\left|\nabla_{\Gamma} w\right|^{2}+\left|\frac{\partial w}{\partial \nu}\right|^{2}
$$

so that

$$
\nu_{n}|\nabla w|^{2}-2 \operatorname{Re}\left(\frac{\partial \bar{w}}{\partial x_{n}} \frac{\partial w}{\partial \nu}\right)=\nu_{n}\left|\nabla_{\Gamma} w\right|^{2}-\nu_{n}\left|\frac{\partial w}{\partial \nu}\right|^{2}-2 \operatorname{Re}\left(\left(e_{n} \cdot \nabla_{\Gamma} \bar{w}\right) \frac{\partial w}{\partial \nu}\right)
$$


Rearranging terms and noting that $\partial w / \partial \nu=i k \beta w$ on $\operatorname{supp}\left(\phi_{A}(r)\right) \cap \Gamma$, we find that

$$
\begin{aligned}
& 2 \int_{S_{H}} \phi_{A}(r)\left|\frac{\partial w}{\partial x_{n}}\right|^{2} d x-\int_{\Gamma} \phi_{A}(r)\left(H-x_{n}\right) \nu_{n}\left|\nabla_{\Gamma} w\right|^{2} d s \\
& =\int_{S_{H}}\left\{\phi_{A}(r)\left(|\nabla w|^{2}-k^{2}|w|^{2}\right)-2 \phi_{A}^{\prime}(r)\left(x_{n}-H\right) \operatorname{Re}\left(\frac{\partial \bar{w}}{\partial x_{n}} \frac{\partial w}{\partial r}\right)\right\} d x \\
& \quad-2 \operatorname{Re} \int_{S_{H}} \phi_{A}(r)\left(x_{n}-H\right) g \frac{\partial \bar{w}}{\partial x_{n}} d x-\int_{\Gamma}\left(H-x_{n}\right) \phi_{A}(r) \nu_{n} k^{2}\left(1+|\beta|^{2}\right)|w|^{2} d s \\
& \quad-2 k \int_{\Gamma}\left(H-x_{n}\right) \phi_{A}(r) \operatorname{Re}\left(\left(e_{n} \cdot \nabla_{\Gamma} \bar{w}\right) i \beta w\right) d s
\end{aligned}
$$

Now, where $L^{\prime}=\sqrt{1+L^{2}}$,

$$
-e_{n} \cdot \nu=-\nu_{n} \geq \frac{1}{L^{\prime}}
$$

and

$$
\left|e_{n} \cdot \nabla_{\Gamma} w\right| \leq \frac{L}{L^{\prime}}\left|\nabla_{\Gamma} w\right|
$$

So

$$
\begin{aligned}
& \left|2 k \int_{\Gamma}\left(H-x_{n}\right) \phi_{A}(r) \operatorname{Re}\left(\left(e_{n} \cdot \nabla_{\Gamma} \bar{w}\right) i \beta w\right) d s\right| \\
& \leq \frac{2 k L}{L^{\prime}} \int_{\Gamma} \phi_{A}(r)\left|\nabla_{\Gamma} w\right| B|w|\left(H-x_{n}\right) d s \\
& \leq \frac{1}{L^{\prime}} \int_{\Gamma} \phi_{A}(r)\left|\nabla_{\Gamma} w\right|^{2}\left(H-x_{n}\right) d s+\frac{k^{2} L^{2}}{L^{\prime}} \int_{\Gamma} \phi_{A}(r)\left(H-x_{n}\right) B^{2}|w|^{2} d s
\end{aligned}
$$

while

$$
\begin{aligned}
\left|2 \operatorname{Re} \int_{S_{H}} \phi_{A}(r)\left(H-x_{n}\right) g \frac{\partial \bar{w}}{\partial x_{n}} d x\right| \leq & \int_{S_{H}} \phi_{A}(r)\left|\frac{\partial w}{\partial x_{n}}\right|^{2} d x \\
& +\int_{S_{H}} \phi_{A}(r)\left(H-x_{n}\right)^{2}|g|^{2} d x .
\end{aligned}
$$

Combining (43), (46), (47) and noting (44) we have

$$
\begin{aligned}
& \int_{S_{H}} \phi_{A}(r)\left|\frac{\partial w}{\partial x_{n}}\right|^{2} d x \\
& \leq \int_{S_{H}}\left\{\phi_{A}(r)\left(|\nabla w|^{2}-k^{2}|w|^{2}\right)-2 \phi_{A}^{\prime}(r)\left(x_{n}-H\right) \operatorname{Re}\left(\frac{\partial \bar{w}}{\partial x_{n}} \frac{\partial w}{\partial r}\right)\right\} d x \\
& \quad+\int_{S_{H}} \phi_{A}(r)\left(H-x_{n}\right)^{2}|g|^{2} d x \\
& \quad-\int_{\Gamma}\left(H-x_{n}\right) \phi_{A}(r) \nu_{n} k^{2}\left(1+B^{2}\right)|w|^{2} d s+\frac{k^{2} L^{2}}{L^{\prime}} \int_{\Gamma}\left(H-x_{n}\right) \phi_{A}(r) B^{2}|w|^{2} d s
\end{aligned}
$$

We now wish to let $A \rightarrow \infty$. The only problem is the term involving $\phi_{A}^{\prime}$ which we estimate as follows. Let $S_{H}^{b}=\left\{x \in S_{H}:|\tilde{x}|<b\right\}$ for $b \geq 1$. Then

$$
\left|\int_{S_{H}}\left\{2 \phi_{A}^{\prime}(r)\left(x_{n}-H\right) \operatorname{Re}\left(\frac{\partial \bar{w}}{\partial x_{n}} \frac{\partial w}{\partial r}\right)\right\} d x\right| \leq 2 M\left(H-f_{-}\right) \int_{S_{H}^{A+1} \backslash \overline{S_{H}^{A}}}|\nabla w|^{2} d x \rightarrow 0
$$


as $A \rightarrow \infty$, where the convergence follows from the fact that $w \in H^{1}\left(S_{H}\right)$. Now letting $A \rightarrow \infty$ and using Lebesgue's dominated convergence theorem gives,

$$
\begin{aligned}
\int_{S_{H}}\left|\frac{\partial w}{\partial x_{n}}\right|^{2} d x \leq & \int_{S_{H}}\left(|\nabla w|^{2}-k^{2}|w|^{2}\right)+\int_{S_{H}}\left(H-x_{n}\right)^{2}|g|^{2} d x \\
& -\int_{\Gamma}\left(H-x_{n}\right) \nu_{n} k^{2}\left(1+B^{2}\right)|w|^{2} d s+\frac{k^{2} L^{2}}{L^{\prime}} \int_{\Gamma}\left(H-x_{n}\right) B^{2}|w|^{2} d s .
\end{aligned}
$$

Use of (40) leads to

$$
\begin{aligned}
\int_{S_{H}}\left|\frac{\partial w}{\partial x_{n}}\right|^{2} d x \leq & \sec \Phi\|g\|_{2}\|w\|_{2}+\frac{\kappa^{2}}{k^{2}}\|g\|_{2}^{2} \\
& +k \kappa \int_{\Gamma}\left|\nu_{n}\right|\left(1+B^{2}\right)|w|^{2} d s+\frac{k \kappa L^{2}}{L^{\prime}} \int_{\Gamma} B^{2}|w|^{2} d s \\
\leq & \sec \Phi\|g\|_{2}\|w\|_{2}+\frac{\kappa^{2}}{k^{2}}\|g\|_{2}^{2}+k \kappa \int_{\Gamma}\left[1+B^{2}\left(1+\frac{L^{2}}{L^{\prime}}\right)\right]|w|^{2} d s \\
\leq & \sec \Phi\|g\|_{2}\|w\|_{2}+\frac{\kappa^{2}}{k^{2}}\|g\|_{2}^{2}+k \kappa\left[1+B^{2}(1+L)\right] \int_{\Gamma}|w|^{2} d s .
\end{aligned}
$$

Combining (42) and (50) gives

$$
k^{2}\|w\|_{2}^{2} \leq \kappa^{2} \sec \Phi\|g\|_{2}\|w\|_{2}+\frac{\kappa^{4}}{k^{2}}\|g\|_{2}^{2}+k \kappa\left[2+\kappa^{2}\left(1+B^{2}(1+L)\right)\right] \int_{\Gamma}|w|^{2} d s .
$$

Using (41) we get

$$
\begin{aligned}
k^{2}\|w\|_{2}^{2} & \leq\left[\kappa^{2} \sec \Phi+\frac{2 \kappa+\kappa^{3}\left(1+B^{2}(1+L)\right)}{\eta}\right]\|g\|_{2}\|w\|_{2}+\frac{\kappa^{4}}{k^{2}}\|g\|_{2}^{2} \\
& \leq \frac{1}{2} k^{2}\|w\|_{2}^{2}+\frac{\kappa^{2}}{k^{2}}\left[\kappa^{2}+\frac{1}{2}\left[\kappa \sec \Phi+\frac{2+\kappa^{2}\left(1+B^{2}(1+L)\right)}{\eta}\right]^{2}\right]\|g\|_{2}^{2},
\end{aligned}
$$

so that, using $\sqrt{a^{2}+b^{2}} \leq a+b$, for $a, b>0$,

$$
\begin{aligned}
k\|w\|_{2} & \leq \frac{\kappa}{k}\left[2 \kappa^{2}+\left[\kappa \sec \Phi+\frac{2+\kappa^{2}\left(1+B^{2}(1+L)\right)}{\eta}\right]^{2}\right]^{\frac{1}{2}}\|g\|_{2} \\
& \leq \frac{\kappa}{k}\left[\sqrt{2} \kappa+\left[\kappa \sec \Phi+\frac{2+\kappa^{2}\left(1+B^{2}(1+L)\right)}{\eta}\right]\right]\|g\|_{2} .
\end{aligned}
$$

Defining

$$
F:=\kappa\left[\kappa[\sqrt{2}+\sec \Phi]+\frac{2+\kappa^{2}\left(1+B^{2}(1+L)\right)}{\eta}\right],
$$

and using (40) we get

$$
k^{2}\|w\|_{H^{1}\left(S_{H}\right)}^{2} \leq 2 k^{4}\|w\|_{2}^{2}+\sec \Phi k^{2}\|g\|_{2}\|w\|_{2} \leq\left[2 F^{2}+\sec \Phi F\right]\|g\|_{2}^{2}
$$

so that

$$
k\|w\|_{H^{1}\left(S_{H}\right)} \leq\left(2 \sqrt{2} F+\frac{\sec \Phi}{4 \sqrt{2}}\right)\|g\|_{2} .
$$

Combining Lemmas 15, 14 and 13 with Corollary 12, we have the following result. 
Lemma 16. If $\Gamma$ is given by (3) with $f$ satisfying (2) and with $f \in C^{\infty}\left(\mathbf{R}^{n-1}\right)$ and $\beta \in C^{\infty}(\Gamma) \cap L^{\infty}(\Gamma)$ such that (A2) holds, then the variational problem (17) has a unique solution $u \in H^{1}\left(S_{H}\right)$ for every $\mathcal{G} \in H^{1}\left(S_{H}\right)^{*}$ and the solution satisfies the estimate (30).

Before we extend Lemma 16 to non-smooth surfaces we will need two preliminary and standard lemmas. The first concerns approximation of a Lipschitz function by smooth Lipschitz functions. The proof can be found in [4].

Lemma 17. Let $f: \mathbf{R}^{n-1} \rightarrow \mathbf{R}$ be a Lipschitz function with Lipschitz constant $L$. Then for all $\epsilon>0$, there exists $f_{\epsilon}: \mathbf{R}^{n-1} \rightarrow \mathbf{R}$ such that

i) $f_{\epsilon} \in C^{\infty}\left(\mathbf{R}^{n-1}\right)$,

ii) $f_{\epsilon}$ is Lipschitz and $\left|f_{\epsilon}(\tilde{x})-f_{\epsilon}(\tilde{y})\right| \leq L|\tilde{x}-\tilde{y}|, \tilde{x}, \tilde{y} \in \mathbf{R}^{n-1}$,

iii) $f_{\epsilon} \geq f+\epsilon / 6$

iv) $\left\|f_{\epsilon}-f\right\|_{L^{\infty}\left(\mathbf{R}^{n-1}\right)}<\epsilon$,

v) for $i \in\{1, \ldots, n-1\}$, $\tilde{\epsilon}>0$, and compact $K \subset \mathbf{R}^{n-1}$,

$$
\left\|\frac{\partial f}{\partial x_{i}}-\frac{\partial f_{\epsilon}}{\partial x_{i}}\right\|_{L^{p}(K)}<\tilde{\epsilon},
$$

for $1<p<\infty$, provided $\epsilon$ is sufficiently small.

In the next lemma we extend, by reflection, a test function onto a larger domain. We will not make use of standard extension theorems because we need to know explicit bounds. Again, the proof can be found in [4].

Lemma 18. Let $H \geq f_{+}+\mu$ and suppose $\Gamma$ is given by (3) with $f$ Lipschitz with Lipschitz constant L. Let $f^{*} \in C^{\infty}\left(\mathbf{R}^{n-1}\right)$ be such that, for $\tilde{x}, \tilde{y} \in \mathbf{R}^{n-1}$,

$$
\left|f^{*}(\tilde{x})-f^{*}(\tilde{y})\right| \leq L|\tilde{x}-\tilde{y}|, \quad f^{*}(\tilde{x}) \geq f(\tilde{x}),
$$

and such that

$$
f^{*}(\tilde{x})+\left(f^{*}(\tilde{x})-f(\tilde{x})\right)<H .
$$

Let $S_{H}^{*}=D^{*} \backslash \overline{U_{H}}$, where $D^{*}$ is the epigraph of $f^{*}$. Then, for all $v \in \mathcal{D}\left(S_{H}^{*}\right)$, we can extend $v$ to a function on $S_{H}$ such that $v \in H^{1}\left(S_{H}\right),\left.v\right|_{S_{H} \backslash S_{H}^{*}} \in \mathcal{D}\left(S_{H} \backslash \overline{S_{H}^{*}}\right)$ and

$$
\|v\|_{H^{1}\left(S_{H} \backslash \overline{S_{H}^{*}}\right)} \leq 2 \sqrt{\left(1+4(n-1) L^{2}\right)}\|v\|_{H^{1}\left(S_{H}^{*}\right)} .
$$

We next show that Lemmas 15 and 16 hold for domains with boundaries given by arbitrary Lipschitz graphs.

Lemma 19. Suppose $\Gamma$ is given by (3) with $f$ Lipschitz with Lipschitz constant $L, H \geq f_{+}+\mu, g \in L^{2}\left(S_{H}\right)$, and $\beta \in C(\Gamma) \cap L^{\infty}(\Gamma)$ is such that $\beta$ is the restriction to $\Gamma$ of

$$
\beta^{*} \in C^{\infty}\left(\mathbf{R}^{n}\right) \cap L^{\infty}\left(\mathbf{R}^{n}\right) \text { such that } \frac{\partial \beta^{*}}{\partial x_{n}}=0, \text { and such that } \operatorname{Re}\left(\beta^{*}\right) \geq \eta>0 .
$$

Suppose that $w \in H^{1}\left(S_{H}\right)$ satisfies

$$
c(w, v)=-(g, v), \quad v \in H^{1}\left(S_{H}\right) .
$$

Then

$$
k\|w\|_{H^{1}\left(S_{H}\right)} \leq E\|g\|_{2}
$$

where $E$ is given by (31). 
Proof. Fix a sequence $\epsilon_{m} \rightarrow 0$ such that $\epsilon_{m+1}<\epsilon_{m} / 6$, for $m \in \mathbf{N}$. By Lemma 17, there exists a sequence of Lipschitz functions $f_{\epsilon_{m}} \in C^{\infty}\left(\mathbf{R}^{n-1}\right)$, with Lipschitz constant $L$, such that $\left\|f-f_{\epsilon_{m}}\right\|_{L^{\infty}\left(\mathbf{R}^{n-1}\right)}<\epsilon_{m}$, such that $f_{\epsilon_{m}} \geq f+\epsilon_{m} / 6$, and we may assume that $2 f_{\epsilon_{m}}-f<H$ for all $m \in \mathbf{N}$. Note that the $f_{\epsilon_{m}}$ are decreasing. For each $m \in \mathbf{N}$, let $D_{m} \subseteq \mathbf{R}^{n}$, denote the epigraph of $f_{\epsilon_{m}}$, let $S_{H}^{m}=D_{m} \backslash \overline{U_{H}}$ and let $\Gamma_{m}=\partial D_{m}$.

Let $c_{m}: H^{1}\left(S_{H}^{m}\right) \times H^{1}\left(S_{H}^{m}\right) \rightarrow \mathbf{C}$, be defined by (15) with $S_{H}, \Gamma$ replaced by $S_{H}^{m}, \Gamma_{m}$ and $\beta$ replaced by $\beta^{*}$.

Fix $m \in \mathbf{N}$. Every $v \in \mathcal{D}\left(S_{H}^{m}\right)$ can be extended to an element of $H^{1}\left(S_{H}\right)$ by Lemma 18 such that

$$
\|v\|_{H^{1}\left(S_{H} \backslash \overline{S_{H}^{m}}\right)} \leq 2 \sqrt{\left(1+4(n-1) L^{2}\right)}\|v\|_{H^{1}\left(S_{H}^{m}\right)} .
$$

Now, let $v \in \mathcal{D}\left(S_{H}^{m}\right)$, fix $\delta>0$ and choose $w_{k} \in \mathcal{D}\left(S_{H}\right)$ such that $\left\|w-w_{k}\right\|_{H^{1}\left(S_{H}\right)}<$ $\delta$. Then

$$
\begin{aligned}
c_{m}\left(w_{k}, v\right)= & \int_{S_{H}^{m}} \nabla w_{k} \cdot \nabla \bar{v}-k^{2} w_{k} \bar{v} d x+\int_{\Gamma_{H}} \bar{v} T \gamma_{-} w_{k} d s-\int_{\Gamma_{m}} i k \beta^{*} w_{k} \bar{v} d s \\
= & c\left(w_{k}, v\right)-\int_{S_{H} \backslash \overline{S_{H}^{m}}} \nabla w_{k} \cdot \nabla \bar{v}-k^{2} w_{k} \bar{v} d x+\int_{\Gamma} i k \beta w_{k} \bar{v} d s \\
& -\int_{\Gamma_{m}} i k \beta^{*} w_{k} \bar{v} d s \\
= & c(w, v)+c\left(w_{k}-w, v\right)-\int_{S_{H} \backslash \overline{S_{H}^{m}}} \nabla w_{k} \cdot \nabla \bar{v}-k^{2} w_{k} \bar{v} d x+\int_{\Gamma} i k \beta w_{k} \bar{v} d s \\
& -\int_{\Gamma_{m}} i k \beta^{*} w_{k} \bar{v} d s \\
= & -\int_{S_{H}^{m}} g \bar{v} d x+c\left(w_{k}-w, v\right)-\int_{S_{H} \backslash \overline{S_{H}^{m}}} \nabla w_{k} . \nabla \bar{v}-k^{2} w_{k} \bar{v}+g \bar{v} d x \\
& +\int_{\Gamma} i k \beta w_{k} \bar{v} d s-\int_{\Gamma_{m}} i k \beta^{*} w_{k} \bar{v} d s .
\end{aligned}
$$

Now define $\mathcal{H}_{m}: \mathcal{D}\left(S_{H}^{m}\right) \rightarrow \mathbf{C}$ by

(58) $\mathcal{H}_{m}(v):=-\int_{S_{H} \backslash \overline{S_{H}^{m}}} \nabla w_{k} \cdot \nabla \bar{v}-k^{2} w_{k} \bar{v}+g \bar{v} d x+\int_{\Gamma} i k \beta w_{k} \bar{v} d s-\int_{\Gamma_{m}} i k \beta^{*} w_{k} \bar{v} d s$.

To show that $\mathcal{H}_{m}$ defines a continuous anti-linear functional on $H^{1}\left(S_{H}^{m}\right)$, we first of all note that

$$
\begin{aligned}
& \left|\int_{S_{H} \backslash \overline{S_{H}^{m}}} g \bar{v}+\nabla w_{k} \cdot \nabla \bar{v}-k^{2} w_{k} \bar{v} d x\right| \\
& \leq\left(k^{-1}\|g\|_{L^{2}\left(S_{H} \backslash \overline{S_{H}^{m}}\right)}+\left\|\nabla w_{k}\right\|_{L^{2}\left(S_{H} \backslash \overline{S_{H}^{m}}\right)}+k\left\|w_{k}\right\|_{L^{2}\left(S_{H} \backslash \overline{S_{H}^{m}}\right)}\right) \\
& \quad \cdot 2 \sqrt{\left(1+4(n-1) L^{2}\right)}\|v\|_{H^{1}\left(S_{H}^{m}\right)}
\end{aligned}
$$

using (54).

To estimate the second term on the right hand side of (58), define $h: S_{H} \backslash S_{H}^{m} \rightarrow \mathbf{R}$ by $h\left(\tilde{x}, x_{n}\right)=J_{f}(\tilde{x})=\sqrt{1+|\nabla f(\tilde{x})|^{2}}$ for all $\tilde{x}$ at which $f$ is 
differentiable. In addition let $K=\operatorname{supp}\left(w_{k}\right)$, let $\|\cdot\|$ denote $\|\cdot\|_{L^{\infty}\left(\mathbf{R}^{n}\right)}$, and let

$$
l(\tilde{x})=\left(\int_{\mathbf{R}^{n-1} \cap K}\left|J_{f_{\epsilon}}(\tilde{x})-J_{f}(\tilde{x})\right|^{2}\right)^{\frac{1}{2}} .
$$

Then

$$
\begin{aligned}
& \left|\int_{\Gamma_{m}} i k \beta^{*} w_{k} \bar{v} d s-\int_{\Gamma} i k \beta w_{k} \bar{v} d s\right| \\
& =\left|\int_{\mathbf{R}^{n-1}} J_{f_{\epsilon_{m}}}(\tilde{x}) i k \beta^{*} w_{k} \bar{v}\left(\tilde{x}, f_{\epsilon_{m}}(\tilde{x})\right) d \tilde{x}-\int_{\mathbf{R}^{n-1}} J_{f}(\tilde{x}) i k \beta w_{k} \bar{v}(\tilde{x}, f(\tilde{x})) d \tilde{x}\right| \\
& \leq\left|\int_{\mathbf{R}^{n-1}} J_{f}(\tilde{x}) \int_{f(\tilde{x})}^{f_{\epsilon_{m}}(\tilde{x})} \frac{\partial}{\partial x_{n}}\left(i k \beta^{*} w_{k} \bar{v}\right)(x) d x_{n} d \tilde{x}\right| \\
& +\left|\int_{\mathbf{R}^{n-1} \cap K}\left(J_{f_{\epsilon_{m}}}(\tilde{x})-J_{f}(\tilde{x})\right) i k \beta^{*} w_{k} \bar{v}\left(\tilde{x}, f_{\epsilon_{m}}(\tilde{x})\right) d \tilde{x}\right| \\
& \leq \sqrt{1+L^{2}}\left\{\left(\int_{S_{H} \backslash S_{H}^{m}} k^{2}\left|\beta^{*}\right|^{2}\left|w_{k}\right|^{2} d x\right)^{\frac{1}{2}}\left(\int_{S_{H} \backslash S_{H}^{m}}\left|\frac{\partial v}{\partial x_{n}}\right|^{2} d x\right)^{\frac{1}{2}}\right. \\
& \left.+\left(\int_{S_{H} \backslash S_{H}^{m}}\left|\beta^{*}\right|^{2}\left|\frac{\partial w_{k}}{\partial x_{n}}\right|^{2} d x\right)^{\frac{1}{2}}\left(\int_{S_{H} \backslash S_{H}^{m}} k^{2}|v|^{2} d x\right)^{\frac{1}{2}}\right\} \\
& +l(\tilde{x})\left(\int_{\mathbf{R}^{n-1}}\left|i k \beta^{*} w_{k} \bar{v}\left(\tilde{x}, f_{\epsilon_{m}}(\tilde{x})\right)\right|^{2} d \tilde{x}\right)^{\frac{1}{2}} \\
& \leq \sqrt{1+L^{2}}\left\{k\left\|\beta^{*}\right\|\left(\int_{S_{H} \backslash S_{H}^{m}}\left|w_{k}\right|^{2} d x\right)^{\frac{1}{2}}+\left\|\beta^{*}\right\|\left(\int_{S_{H} \backslash S_{H}^{m}}\left|\frac{\partial w_{k}}{\partial x_{n}}\right|^{2} d x\right)^{\frac{1}{2}}\right\} \\
& \cdot \sqrt{2\left(1+4(n-1) L^{2}\right)}\|v\|_{H^{1}\left(S_{H}^{m}\right)} \\
& +l(\tilde{x})\left\|\beta^{*}\right\|\left\|w_{k}\right\| k^{\frac{1}{2}} \sqrt{\sqrt{1+L^{2}}\left(1+\frac{2}{k \mu}\right)}\|v\|_{H^{1}\left(S_{H}^{m}\right)},
\end{aligned}
$$

using Lemma 5 and assuming that $S_{H}^{m}$ is an $(L, \mu / 2,1)$ Lipschitz domain, which it is, provided $\epsilon_{m}<\mu / 2$.

We may now write $(57)$ as

$$
c_{m}\left(w_{k}, v\right)=-\int_{S_{H}^{m}} g \bar{v} d x+c\left(w_{k}-w, v\right)+\mathcal{H}_{m}(v), \quad v \in \mathcal{D}\left(S_{H}^{m}\right) .
$$

By the density of $\mathcal{D}\left(S_{H}^{m}\right)$ in $H^{1}\left(S_{H}^{m}\right)$, and the continuity of $c_{m}, c$ and $\mathcal{H}_{m},(62)$ must hold for all $v \in H^{1}\left(S_{H}^{m}\right)$. Since $\Gamma_{m} \in C^{\infty}\left(\mathbf{R}^{n-1}\right)$ and $\beta^{*} \in C^{\infty}\left(\Gamma_{m}\right)$ then by Lemma 16 there exist unique $w^{\prime}, w^{\prime \prime}$ such that

$$
c_{m}\left(w^{\prime}, v\right)=-\int_{S_{H}^{m}} g \bar{v} d x, \quad c_{m}\left(w^{\prime \prime}, v\right)=c\left(w_{k}-w, v\right)+\mathcal{H}_{m}(v)
$$

and by Lemmas 15 and 16

$$
\begin{aligned}
\left\|w^{\prime}\right\|_{H^{1}\left(S_{H}^{m}\right)} & \leq k^{-1} E\|g\|_{L^{2}\left(S_{H}^{m}\right)}, \\
\left\|w^{\prime \prime}\right\|_{H^{1}\left(S_{H}^{m}\right)} & \leq \sec \Phi(1+2 E)\left\{\left\|\mathcal{H}_{m}\right\|_{H^{1}\left(S_{H}^{m}\right)^{*}}+\|c\|\left\|w_{k}-w\right\|_{H^{1}\left(S_{H}\right)}\right\} .
\end{aligned}
$$


By Lemma 17 and the fact that $\partial \beta^{*} / \partial x_{n}=0, E$ and $\sec \Phi$ are independent of $m$. Clearly $w_{k}=w^{\prime}+w^{\prime \prime}$. So

$$
\begin{aligned}
\left\|w_{k}\right\|_{H^{1}\left(S_{H}^{m}\right)} \leq & k^{-1} E\|g\|_{L^{2}\left(S_{H}^{m}\right)} \\
& +\sec \Phi(1+2 E)\left\{\left\|\mathcal{H}_{m}\right\|_{H^{1}\left(S_{H}^{m}\right)^{*}}+\|c\|\left\|w_{k}-w\right\|_{H^{1}\left(S_{H}\right)}\right\} .
\end{aligned}
$$

Now let $m \rightarrow \infty$, using (61) to estimate $\left\|\mathcal{H}_{m}\right\|_{H^{1}\left(S_{H}^{m}\right)^{*}}$, using Lebesgue's monotone convergence theorem to show that the terms $\|\cdot\|_{L^{2}\left(S_{H} \backslash \overline{S_{H}^{m}}\right)} \rightarrow 0$, and using Lemma 17 part v) we see that

$$
\left\|w_{k}\right\|_{H^{1}\left(S_{H}\right)} \leq k^{-1} E\|g\|_{2}+\sec \Phi(1+2 E)\|c\| \delta .
$$

Finally arbitrariness of $\delta>0$ gives the result.

Combining Lemmas 19, 14 and 13 with Corollary 12, we have the following result.

Lemma 20. If $\Gamma$ is given by (3) with $f$ Lipschitz with Lipschitz constant L, and $\beta \in C(\Gamma) \cap L^{\infty}(\Gamma)$ satisfies the hypotheses of Lemma 19, then the variational problem (17) has a unique solution $u \in H^{1}\left(S_{H}\right)$ for every $\mathcal{G} \in H^{1}\left(S_{H}\right)^{*}$ and the solution satisfies the estimate (30).

We now show that Lemmas 19 and 20 hold for more general $\beta \in L^{\infty}(\Gamma)$.

Lemma 21. Suppose $\Gamma$ is given by (3) with $f$ Lipschitz with Lipschitz constant L. Let $H \geq f_{+}+\mu, g \in L^{2}\left(S_{H}\right)$, and $\beta \in L^{\infty}(\Gamma)$ be such that assumption (A2) holds, and suppose $w \in H^{1}\left(S_{H}\right)$ satisfies

$$
b(w, v)=-(g, v), \quad v \in H^{1}\left(S_{H}\right) .
$$

Then

where $E$ is given by (31).

$$
k\|w\|_{H^{1}\left(S_{H}\right)} \leq E\|g\|_{2}
$$

Proof. For $\delta>0$ let $\psi_{\delta} \in C_{0}^{\infty}\left(\mathbf{R}^{n}\right)$ be such that $\psi_{\delta}>0, \psi_{\delta}(x)=0$ if $|x|>\delta$, and such that $\int_{\mathbf{R}^{n}} \psi_{\delta}(x) d x=1$ Then define, $\beta_{\delta} \in C^{\infty}\left(\mathbf{R}^{n-1}\right)$ by

$$
\beta_{\delta}(\tilde{x})=\int_{\mathbf{R}^{n-1}} \beta(\tilde{x}-\tilde{y}, f(\tilde{x}-\tilde{y})) \psi_{\delta}(\tilde{y}) d \tilde{y}
$$

and then extend $\beta_{\delta}$ to a function $\beta_{\delta} \in C^{\infty}\left(\mathbf{R}^{n}\right)$ via $\beta_{\delta}\left(\tilde{x}, x_{n}\right)=\beta_{\delta}(\tilde{x})$. It follows that $\beta_{\delta} \in C(\Gamma)$ and that $\beta_{\delta}$ is the restriction to $\Gamma$ of a function $\beta_{\delta} \in C^{\infty}\left(\mathbf{R}^{n}\right)$ such that $\partial \beta_{\delta} / \partial x_{n}=0$. Note that, for $\tilde{x} \in \mathbf{R}^{n-1}$,

$$
\begin{aligned}
\operatorname{Re}\left(\beta_{\delta}(\tilde{x})\right) & =\operatorname{Re} \int_{\mathbf{R}^{n-1}} \beta(\tilde{x}-\tilde{y}, f(\tilde{x}-\tilde{y})) \psi_{\delta}(\tilde{x}) d \tilde{x} \\
& =\int_{\mathbf{R}^{n-1}} \psi_{\delta}(\tilde{x}) \operatorname{Re} \beta(\tilde{x}-\tilde{y}, f(\tilde{x}-\tilde{y})) d \tilde{x} \geq \eta
\end{aligned}
$$

and

$$
\left|\beta_{\delta}(\tilde{x})\right| \leq \int_{\mathbf{R}^{n-1}} \psi_{\delta}(\tilde{x})|\beta(\tilde{x}-\tilde{y}, f(\tilde{x}-\tilde{y}))| d \tilde{x} \leq B \Longrightarrow\left\|\beta_{\delta}\right\|_{L^{\infty}\left(\mathbf{R}^{n-1}\right)} \leq B .
$$

Further, since $\operatorname{Re}\left(e^{-i(\pi / 2+\Phi)} \beta\right) \geq 0$, it follows, by arguing as above, that $\operatorname{Re}\left(e^{-i(\pi / 2+\Phi)}\right.$ $\left.\cdot \beta_{\delta}\right) \geq 0$. This ensures that $\Phi_{\delta}:=\min \left\{0, \inf _{x \in \mathbf{R}^{n}} \arg \beta_{\delta}\right\} \geq \Phi$, which in turn means that $\sec \Phi_{\delta} \leq \sec \Phi$. 
Fix $\epsilon>0$, and choose $w_{m} \in \mathcal{D}\left(S_{H}\right)$ such that $\left\|w_{m}-w\right\|_{H^{1}\left(S_{H}\right)}<\epsilon$. Standard arguments (e.g. [44, Theorem 3.4]) show that $\beta_{\delta} \rightarrow \beta$ in the normed space $L^{2}\left(\operatorname{supp}\left(w_{m}\right) \cap \Gamma\right)$. Thus if we choose $\delta$ sufficiently small then

$$
\left(\int_{\Gamma} k\left|\beta_{\delta}(s)-\beta(s)\right|^{2}\left|w_{m}(s)\right|^{2} d s\right)^{\frac{1}{2}}<\sqrt{k}\left\|w_{m}\right\|_{L^{\infty}(\Gamma)}\left\|\beta_{\delta}-\beta\right\|_{L^{2}\left(\operatorname{supp}\left(w_{m}\right) \cap \Gamma\right)}<\epsilon .
$$

Now

$$
c\left(w_{m}, v\right)=-(g, v)+c\left(w_{m}-w, v\right), \quad v \in H^{1}\left(S_{H}\right),
$$

so that, for $v \in H^{1}\left(S_{H}\right)$,

$$
\begin{aligned}
& \int_{S_{H}} \nabla w_{m} \cdot \nabla \bar{v}-k^{2} w_{m} \bar{v} d x+\int_{\Gamma_{H}} \gamma_{-} \bar{v} T w_{m} d s-\int_{\Gamma} i k \beta_{\delta} w_{m} \gamma_{*} \bar{v} d s \\
& =-\int_{S_{H}} g \bar{v} d x+c\left(w_{m}-w, v\right)-\int_{\Gamma} i k\left(\beta_{\delta}-\beta\right) w_{m} \gamma_{*} \bar{v} d s .
\end{aligned}
$$

Since $\beta_{\delta}$ satisfies the hypotheses of Lemma 19, then, by Lemmas 20, 19 and 5, (cf. proof of Lemma 19) we obtain

$$
\left\|w_{m}\right\|_{H^{1}\left(S_{H}\right)} \leq k^{-1} E\|g\|_{2}+\sec \Phi(1+2 E)\left[\|c\| \epsilon+\epsilon \sqrt{\sqrt{1+L^{2}}\left(1+\frac{1}{k \mu}\right)}\right],
$$

and the result follows by arbitrariness of $\epsilon>0$.

Theorem 10 now follows by combining Lemmas 21, 14 and 13 with Corollary 12.

\section{References}

[1] Adams, R. A.: Sobolev spaces. - Academic Press, New York, 1975.

[2] Arens, T., and T. Hohage: On radiation conditions for rough surface scattering problems. - IMA J. Appl. Math. 70, 2005, 839-847.

[3] Attenborough, K., I. Bashir, and S. Taherzadeh: Outdoor ground impedance models. J. Acoust. Soc. Amer. 129, 2011, 2806-2819.

[4] BADEn-Riess, T.: Existence, uniqueness and explicit bounds for scattering by rough surfaces. - PhD Thesis, University of Reading, arXiv:1904.04011, 2006.

[5] BAO, G., G. Hu, and T. YIN: Time-harmonic acoustic scattering from locally perturbed half-planes. - SIAM J. Appl. Math. 78:5, 2018, 2672-2691.

[6] Bonnet-Bendhia, A.S., and P. Starling: Guided waves by electromagnetic gratings and non-uniqueness examples for the diffraction problem. - Math. Methods Appl. Sci. 17, 1994, $305-338$.

[7] Chandler-Wilde, S. N.: Some uniform stability and convergence results for integral equations on the real line and projection methods for their solution. - IMA J. Num. Anal. 13, 1993, $509-535$.

[8] Chandler-Wilde, S. N., and C. R. Ross: Scattering by rough surfaces: the Dirichlet problem for the Helmholtz equation in a non-locally perturbed half-plane. - Math. Methods Appl. Sci. 19, 1996, 959-976.

[9] Chandler-Wilde, S. N.: The impedance boundary value problem for the Helmholtz equation in a half-plane. - Math. Methods Appl. Sci. 20, 1997, 813-840.

[10] Chandler-Wilde, S. N., C. R. Ross, and B. Zhang: Scattering by infinite one dimensional rough surfaces. - Proc. R. Soc. Lond. Ser. A 455, 1999, 3767-3787.

[11] Chandler-Wilde, S. N., and A. T. Peplow: A boundary integral equation formulation for the Helmholtz equation in a locally perturbed half-plane. - ZAMM Z. Angew. Math. Mech. 85, $2005,79-88$. 
[12] Chandler-Wilde, S. N., and B. Zhang: A uniqueness result for scattering by infinite rough surfaces. - SIAM J. Appl. Math. 58, 1998, 1774-1790.

[13] Chandler-Wilde, S. N., and J. Elschner: Variational approach in weighted Sobolev spaces to scattering by unbounded rough surfaces. - SIAM J. Math. Anal. 42:6, 2010, 2554-2580.

[14] Chandler-Wilde, S. N., S. Langdon, and L. Ritter: A high-wavenumber boundaryelement method for an acoustic scattering problem. - Philos. Trans. R. Soc. Lond. Ser. A 362, 2004, 647-671.

[15] Chandler-Wilde, S. N., C. R. Ross, and B. Zhang: Scattering by infinite one-dimensional rough surfaces. - Proc. R. Soc. Lond. A Math. 455, 1999, 3767-3787.

[16] Chandler-Wilde, S. N., and B. Zhang: A uniqueness result for scattering by infinite rough surfaces. - SIAM J. Appl. Math. 58, 1998, 1774-1790.

[17] Chandler-Wilde, S. N., E. Heinemeyer, and R. Potthast: Acoustic scattering by mildly rough unbounded surfaces in three dimensions. - SIAM J. Appl. Math. 66, 2006, 1002-1026.

[18] Chandler-Wilde, S. N., E. Heinemeyer, and R. Potthast: A well-posed integral equation formulation for 3D rough surface scattering. - Proc. R. Soc. Lond. Ser. A, 2006.

[19] Chandler-Wilde, S. N., and K. V. Horoshenkov: Padé approximants for the acoustical characteristics of porous media. - J. Acoust. Soc. Amer. 98, 1995, 1119-1129

[20] Chandler-Wilde, S. N., M. Rahman, and C. R. Ross: A fast two-grid and finite section method for a class of integral equations on the real line with application to an acoustic scattering problem in the half-plane. - Numer. Math. 93, 2002, 1-51.

[21] Chandler-Wilde, S. N., and P. Monk: Existence, uniqueness and variational methods for scattering by unbounded rough surfaces. - SIAM J. Math. Anal. 37, 2005, 598-618.

[22] Chandler-Wilde, S. N., and P. Monk: Wave-number explicit bounds in time-harmonic scattering. - SIAM J. Math. Anal. 39, 2008, 1428-1455.

[23] Claeys, X., and H. Haddar: Scattering from infinite rough tubular surfaces. - Math. Methods Appl. Sci. 30:4, 2007, 389-414.

[24] Colton, D. L., and R. Kress: Integral equation methods in scattering theory. - John Wiley, New York, 1983.

[25] DeSanto, J. A.: Scattering by rough surfaces. - In: Scattering and Inverse Scattering in Pure and Applied Science (R. Pike and P. Sabatier, eds.), Academic Press, 2002, 15-36.

[26] Du, K., B. LI, W. Sun, and H. YANG: Electromagnetic scattering from a cavity embedded in an impedance ground plane. - Math. Methods Appl. Sci. 23, 2018.

[27] Durán, M., I. Muga, and J.-C. NÉdÉlec: The Helmholtz equation with impedance in a half plane. - C. R. Acad. Sci. Paris Ser. I 340, 2005, 483-488.

[28] Durán, M., I. Muga, and J.-C. NÉdélec: The Helmholtz equation with impedance in a half space. - C. R. Acad. Sci. Paris Ser. I 341, 2005, 561-566.

[29] Durán, M., I. Muga, and J.-C. NÉDÉLEC: The Helmholtz equation in a locally perturbed half-plane with passive boundary. IMA J. Appl. Math. 71:6, 2006, 853-876.

[30] Elfouhaily, T. M., and C. GuÉRIn: A Critical survey of approximate scattering wave theories from random rough surfaces. - Waves Random Media 14, 2004, R1-R40.

[31] Elschner, J., and G. Hu: Elastic scattering by unbounded rough surfaces: solvability in weighted Sobolev spaces. - Appl. Anal. 94:2, 2015, 251-278.

[32] Elschner, J., and M. Yamamoto: An inverse problem in periodic diffractive optics: reconstruction of Lipschitz grating profiles. - Appl. Anal. 81, 2002, 1307-1328.

[33] GAO, Y., and P. LI: Electromagnetic scattering for time-domain Maxwell's equations in an unbounded structure. - Math. Models Methods Appl. Sci. 27:10, 2017, 1843-1870.

[34] GaO, Y., P. LI, and Y. LI: Analysis of time-domain elastic scattering by an unbounded structure. - Math. Methods Appl. Sci. 41:16, 2018, 7032-7054. 
[35] Hu, G., X. LiU, F. Qu, and B. Zhang: Variational approach to scattering by unbounded rough surfaces with Neumann and generalized impedance boundary conditions. - Commun. Math. Sci. 13, 2015, 511-537.

[36] IhlenburG, F.: Finite element analysis of acoustic scattering. - Appl. Math. Sci. 132, Springer, Berlin, 1998.

[37] KIRsch, A.: Diffraction by periodic structures. - In: Inverse Problems in Mathematical Physics (L. Pävärinta and E. Somersalo, eds.), Springer, Berlin, 1993, 87-102.

[38] Langdon, S., and S. N. Chandler-Wilde: A wavenumber independent boundary element method for an acoustic scattering problem. - SIAM J. Numer. Anal. 43, 2006, 2450-2477.

[39] Lechleiter, A., and S. Ritterbusch: A variational method for wave scattering from penetrable rough layers. - IMA J. Appl. Math. 75, 2010, 366-391.

[40] Lechleiter, A., and R. Zhang: Non-periodic acoustic and electromagnetic, scattering from periodic structures in 3D. - Comput. Math. Appl. 74:11, 2017, 2723-2738.

[41] Li, P., G. Zheng, and W. Zheng: Maxwell's equations in an unbounded structure. - Math. Methods Appl. Sci. 40:3, 2017, 573-588.

[42] Li, P., H. Wu, and W. ZHENG: Electromagnetic scattering by unbounded rough surfaces. SIAM J. Math. Anal. 43:3, 2011, 1205-1231.

[43] LiU, X., B. Zhang, and H. Zhang: A direct imaging method for inverse scattering by unbounded rough surfaces. - SIAM J. Imaging Sci. 11:2, 2018, 1629-1650.

[44] MCLean, W.: Strongly elliptic systems and boundary integral equations. - Cambridge Univ. Press, 2000.

[45] OgiLvy, J. A.: Theory of wave scattering from random rough surfaces. - Adam Hilger, Bristol, 1991.

[46] Peplow, A. T., and S. N. Chandler-Wilde: Noise propagation from a cutting of arbitrary cross-section and impedance. - J. Sound. Vibr. 223, 1999, 355-378.

[47] Petit, R.: Electromagnetic theory of gratings. - Springer, Berlin, 1980.

[48] Pinel, N., and C. Bourlier: Electromagnetic wave scattering from random rough surfaces: asymptotic models. - John Wiley, 2013.

[49] Saillard, M., and A. Sentenac: Rigorous solutions for electromagnetic scattering from rough surfaces. - Waves Random Media 11, 2001, R103-R137.

[50] Strycharz-Szemberg, B.: A direct and inverse scattering transmission problem for periodic inhomogeneous media. - Math. Methods Appl. Sci. 21, 1998, 969-983.

[51] Verchota, G.: Layer potentials and regularity for the Dirchlet problem for Laplace's equation in Lipschitz domains. - J. Funct. Anal. 59, 1984, 572-611.

[52] Voronovich, A. G.: Wave scattering from rough surfaces. 2nd edition. - Springer, Berlin, 1999.

[53] YelkencI, T.: Imaging of rough surfaces having impedance boundary condition by the use of Newton iterative algorithm. - IEEE Geoscience and Remote Sensing Letters 7, 2010, 190-194.

[54] Warnick, K. F., and W. C. Chew: Numerical simulation methods for rough surface scattering. - Waves Random Media 11, 2001, R1-R30.

[55] Wilcox, C.H.: Scattering theory for diffraction gratings. - Appl. Math. Sci. 46, SpringerVerlag, 1984.

[56] Zhang, B., and S. N. Chandler-Wilde: Integral equation methods for scattering by infinite rough surfaces. - Math. Methods Appl. Sci. 26, 2003, 463-488. 